\title{
A topology optimization method based on the level set method for the design of negative permeability dielectric metamaterials
}

\section{$\operatorname{AUTHOR}(S):$}

Otomori, Masaki; Yamada, Takayuki; Izui, Kazuhiro; Nishiwaki, Shinji; Andkjær, Jacob

\section{CITATION:}

Otomori, Masaki ...[et al]. A topology optimization method based on the level set method for the design of negative permeability dielectric metamaterials. Computer Methods in Applied Mechanics and Engineering 2012, 237-240: 192-211

\section{ISSUE DATE:}

2012-09

URL:

http://hdl.handle.net/2433/171650

\section{RIGHT:}

C 2012 Elsevier B.V.; この論文は出版社版でありません。引用の際には 出版社版をご確認ご利用ください。; This is not the published version. Please cite only the published version. 


\title{
A Topology Optimization Method Based on the Level Set Method for the Design of Negative Permeability Dielectric Metamaterials
}

\author{
Masaki Otomori ${ }^{\mathrm{a}, *, * *}$, Takayuki Yamada ${ }^{\mathrm{b}}$, Kazuhiro Izui ${ }^{\mathrm{a}}$, \\ Shinji Nishiwaki ${ }^{a}$, Jacob Andkjær ${ }^{c}$ \\ ${ }^{a}$ Graduate School of Engineering, Kyoto University, Yoshida-honmachi, Sakyo-ku, \\ Kyoto 606-8501, JAPAN \\ ${ }^{\mathrm{b}}$ Graduate School of Engineering, Nagoya University, Furo-cho, Chikusa-ku, \\ Nagoya 464-8603, JAPAN \\ ${ }^{\mathrm{c}}$ Department of Mechanical Engineering, Technical University of Denmark, \\ DK-2800 Lyngby, DENMARK
}

\begin{abstract}
This paper presents a level set-based topology optimization method for the design of negative permeability dielectric metamaterials. Metamaterials are artificial materials that display extraordinary physical properties that are unavailable with natural materials. The aim of the formulated optimization problem is to find optimized layouts of a dielectric material that achieve negative permeability. The presence of grayscale areas in the optimized configurations critically affects the performance of metamaterials, positively as well as negatively, but configurations that contain grayscale areas are highly impractical from an engineering and manufacturing point of view. Therefore, a topology optimization method that can obtain clear optimized configurations is desirable. Here, a level set-based topology optimization method incorporating a fictitious interface energy is applied to a negative permeability dielectric metamaterial design problem. The optimization algorithm uses the finite element method (FEM) for solving the equilibrium and adjoint equations, and design problems are formulated for both two- and three-dimensional cases. First, the level set-based topology optimization method is explained, and the optimization problems for the design of metamaterials are then discussed. Several optimum design examples for the design of dielectric metamaterials that demonstrate negative effective permeability at prescribed frequencies are provided to confirm the utility and validity of the presented method.
\end{abstract}

Key words: Dielectric Metamaterials, Negative Permeability, Topology Optimization, Finite Element Method, Level Set Method, Adjoint Variable Method 


\section{Introduction}

This paper discusses a level set-based topology optimization method for the design of dielectric metamaterials that achieve a negative permeability at desired frequencies. Electromagnetic metamaterials are artificial materials that exhibit extraordinary electromagnetic properties not available in nature, such as a negative refractive index, that is, negative permittivity and permeability. The existence of such materials was first proposed by Veselago [1] in 1968. After Pendry et al. [2][3] and Smith et al. [4] showed that arrangements of splitring resonators that have negative permeability and metallic wires that have negative permittivity can exhibit negative refraction at a certain frequency, considerable research was carried out to investigate the unusual properties of such materials, and develop certain applications, such as cloaking devices [6], waveguides [5], super lenses [7], leaky wave antennas [8], energy harvesting devices [9], and the like. Furthermore, recently, new types of metamaterials that utilize the magnetic and electric resonance phenomena of dielectric materials rather than effects primarily derived from metallic inclusions have been proposed [10-14]. These new dielectric metamaterials are expected to offer advantages due to improved manufacturability and the possibility of achieving isotropic metamaterials that provide advanced functions under no metallic loss.

Holloway et al. [11] showed theoretically that negative effective permittivity and negative effective permeability can be simultaneously achieved with appropriately designed dielectric spheres embedded in a host material. Subsequently, more practical structures based on this approach were suggested, such as structures using dielectric particles of two different radii [12], structures using identically sized spheres but with different values of dielectric constant [13], and arrays of cylindrical dielectric materials [14]. Experimental verifications are provided for three-dimensionally isotropic dielectric metamaterials consisting of an array of dielectric cubes that exhibit negative permeability [15], and an array of dielectric rods [16], and cubic dielectric particles [17] that exhibit negative permittivity and negative permeability simultaneously. Furthermore, applications such as all-dielectric cloaking devices [18][19], as well as waveguides [20] and leaky wave antennas [21] composed of dielectric materials and metallic plates, have been discussed.

Most electromagnetic metamaterials consist of periodic arrays of unit cells that are adequately small compared to the wavelength of the target frequency, with cells composed of a layer of dielectric material, with or without metallic inclusions. The overall structure of such periodic arrays can be considered

\footnotetext{
* Corresponding author. Tel:+81 75753 5198; Fax:+81 757535857

**Email address: otomori.masaki.58r@st.kyoto-u.ac.jp
} 
as an effectively homogeneous electromagnetic structure, so the electromagnetic metamaterial behaves as a material having negative properties exhibited globally, whereas the individual cell materials do not exhibit these properties. Several methods have been proposed to obtain the effective properties of electromagnetic metamaterials, such as homogenization methods based on the asymptotic-expansion approach [22-25] and the energy-based approach [26] that can be applied when the periodic unit cell can be considered as infinitely small compared to the wavelength, a method that computes the effective properties by averaging electric and magnetic fields in the unit cell [27], and methods that extract effective properties from S-parameters, namely, the complex transmission and reflection coefficients[28-31]. Such effective medium theories provide the basis for the design of metamaterial unit cells that can be used to construct useful metamaterials.

Several unit cell layouts have been proposed that achieve good performance at certain desired frequencies [32]. However, the unit cell layout crucially affects the performance of metamaterials, and it is usually difficult or time-consuming to find appropriate unit cell designs by trial and error methods, even for expert engineers. Thus, there is a need for systematic design methods that assist or simplify the design of effective metamaterials. One systematic approach for obtaining desirable unit cell designs is to apply a structural optimization method. Zhou et al. [33] proposed a level set-based structural optimization method for the design of double negative metamaterials, that is, metamaterials with negative permittivity and negative permeability. The aim of the optimization problem in this case was to find an optimized layout of metallic inclusions, and the objective function was formulated using current flow, instead of using the effective permittivity or permeability directly. Subsequently, Zhou et al. [34] proposed a level set-based structural optimization method in which the effective permeability is directly used as an objective function. Concerning the design optimization of metamaterial applications, Yamasaki et al. [35] proposed a level set-based structural optimization method for the design of composite right- and left-handed transmission lines consisting of a metallic waveguide with dielectric inclusions. The aim of optimization problem was to find the optimized configuration of the dielectric inclusion within the unit cell of the transmission line that provides desired dispersion behavior for the composite right- and left-handed transmission line. In level set-based structural optimization methods [36,37], the structural boundaries are represented by the iso-surface of a scalar function called the level set function, and the boundaries are evolved by updating the level set function using a Hamilton-Jacobi equation. However, this method is based on the concept of shape optimization, and only the boundaries of the target structure are changed during the optimization procedure, so topological changes such as the introduction of holes is not allowed, although the number of holes can be decreased during optimization.

On the other hand, topology optimization methods, the most flexible type 
of structural optimization method, allow not only changes in shape, but also topological changes that include increasing the number of holes in the design domain $[38,39]$. Such methods have been applied to a variety of problems, such as stiffness maximization problems [40], eigen-frequency problems [41], electromagnetic problems [42] and, recently, electromagnetic metamaterial problems. Diaz and Sigmund [43] proposed a topology optimization method for the design of negative permeability metamaterials using an S-parameter retrieval method, where the imaginary part of the effective permeability was minimized at a specific frequency, and several designs for metallic structures attached to dielectric substrates that achieved negative permeability were provided. Sigmund [44] proposed a topology optimization method for dielectric metamaterials to obtain dielectric material designs that minimize the effective permeability at a specific frequency, which also employed the S-parameter retrieval method to obtain the effective properties. Choi and Yoo [45] introduced the inverse homogenization method [46] for the design of magnetic materials that demonstrate a desirable prescribed effective permeability value. Zhou et al. [25] proposed an inverse homogenization method for the design of metamaterials, where both permittivity and permeability are simultaneously maximized. El-Kahlout and Kiziltas [47], and Otomori et al. [48] introduced inverse homogenization methods for the design of dielectric materials that demonstrate a desirable prescribed effective permittivity value, using an asymptotic expansion-based homogenization method and Genetic Algorithms (GAs) [47], and an energy-based homogenization method and density-based topology optimization [48], respectively. GAs have also been used to find optimized layouts of a metallic inclusion in the metamaterial unit cell for a negative permeability design problem [49], and for different multi-objective problems [50,51] in which the refractive index and impedance were simultaneously designed [50], and the bandwidth of the negative refractive index was maximized and the dissipation minimized [51].

The basic ideas of topology optimization are (1) the extension of the design domain to a fixed design domain and (2) the replacement of the optimization problem with material distribution problem in the fixed design domain using the characteristic function [52]. Since the characteristic function is a discontinuous function that represents the structure using a value of 0 or 1 , the optimization problem is typically an ill-posed problem. To overcome this difficulty, the Homogenization Design Method (HDM) [38] and density approaches such as the SIMP method [39] have been proposed, where optimized configurations are represented as density distributions, with the density assuming continuous values from 0 to 1 . However, such optimized configurations often include grayscale areas where the density is an intermediate value between 0 and 1. Although configurations including grayscale areas can be considered as composite materials, they are typically impractical to manufacture or meaningless in an engineering sense. To overcome this problem, several kinds of filtering scheme [53-58] have been proposed and applied to many problems, 
to provide optimized configurations that are free from grayscales.

Level set-based topology optimization methods that inherently obtain clear optimized configurations have also been proposed [59,60]. In these methods, the structural boundaries are implicitly represented by the iso-surface of the level set function, so grayscale areas do not appear. Yamada et al. [60] proposed a level set-based topology optimization method where the optimization problem is regularized using the Tikhonov regularization method, and the level set function is updated based on a reaction-diffusion equation. This method not only allows topological changes such as the introduction of holes during the optimization procedure, but also enables the complexity of optimized configurations to be controlled by using appropriate magnitudes of a regularization parameter. The method has been applied to many problems, such as stiffness maximization problems, eigen-frequency problems, compliant mechanism design problems [60], and problems to maximize thermal diffusivity [61], but application to dielectric metamaterial design problems has not yet been reported.

In this paper, the above-mentioned level set-based topology optimization method [60] is applied to the design of negative permeability dielectric metamaterials. The rest of this paper is as follows. Section 2 describes the formulation of the optimization problem for the design of dielectric metamaterials, for both twoand three-dimensional cases, and discusses the level set-based topology optimization method. Section 3 describes the numerical implementation, based on the formulation of the optimization problem, which uses the Finite Element Method (FEM) to solve the electromagnetic wave problem and update the level set function, and the Adjoint Variable Method (AVM) to compute the sensitivity analysis. Finally, several numerical examples are provided for both two- and three-dimensional cases to confirm the validity and utility of the presented method.

\section{Formulations}

\subsection{Governing equation}

\subsubsection{Two-dimensional electromagnetic wave propagation problem}

The design domain for the two-dimensional dielectric metamaterial design problem is illustrated in Fig. 1. Transverse magnetic (TM) waves propagate in $x-y$ direction where the magnetic field vector is polarized orthogonal to the wave direction, and the direction of wave amplitude is in the $z$-direction. Incident waves enter the domain from the left boundary $\Gamma_{1}$ and output waves 
are observed at the right boundary $\Gamma_{2}$. The upper and lower boundaries $\Gamma_{\mathrm{PEC}}$ are set as Perfect Electric Conductors (PEC) under periodic conditions. In the two-dimensional case, the governing equation is the following Helmholtz equation, derived from Maxwell's equation, and the state variable of the governing equation is the magnetic field $H_{z}$ in the $z$ direction. Here, the relative permeability of both the background material and the dielectric material is set to 1 , with air used as the background material.

$$
\nabla \cdot\left(\epsilon_{r}^{-1} \nabla H_{z}\right)+k_{0}^{2} H_{z}=0
$$

where $\epsilon_{r}$ is the relative permittivity and $k_{0}$ is the wave number in a vacuum such that $k_{0}=\omega \sqrt{\epsilon_{0} \mu_{0}}$, where $\omega$ is the angular frequency and $\epsilon_{0}$ and $\mu_{0}$ are the permittivity and permeability in a vacuum, respectively. The boundary conditions are described as follows.

$$
\begin{aligned}
\mathbf{n} \cdot\left(\epsilon_{r}^{-1} \nabla H_{z}\right)+j k_{0} H_{z} & =2 j k_{0} H_{z}^{i} & \text { on } \Gamma_{1} & \\
\mathbf{n} \cdot\left(\epsilon_{r}^{-1} \nabla H_{z}\right)+j k_{0} H_{z} & =0 & & \text { on } \Gamma_{2} \\
\mathbf{n} \cdot\left(\epsilon_{r}^{-1} \nabla H_{z}\right) & =0 & & \text { on } \Gamma_{\mathrm{PEC}}
\end{aligned}
$$

where $\mathbf{n}$ is the normal vector, $j$ is the imaginary unit and $H_{z}^{i}$ is an incident wave. The weak formulation of Eqs.(1)-(4) is then derived as follows.

$$
a_{1}\left(H_{z}, \tilde{H}_{z}\right)+a_{2}\left(H_{z}, \tilde{H}_{z}\right)=l\left(\tilde{H}_{z}\right) \text { for } H_{z} \in U, \quad \tilde{H}_{z} \in U
$$

where

$$
\begin{aligned}
a_{1}\left(H_{z}, \tilde{H}_{z}\right) & =\int_{D} \nabla \tilde{H}_{z} \cdot\left(\epsilon_{r}^{-1} \nabla H_{z}\right) d \Omega-k_{0}^{2} \int_{D} \tilde{H}_{z} H_{z} d \Omega \\
a_{2}\left(H_{z}, \tilde{H}_{z}\right) & =j k_{0} \int_{\Gamma_{1} \cup \Gamma_{2}} \tilde{H}_{z} H_{z} d \Gamma \\
l\left(\tilde{H}_{z}\right) & =2 j k_{0} \int_{\Gamma_{1}} H_{z}^{i} \tilde{H}_{z} d \Gamma \\
U & =\left\{\tilde{H}_{z} \in H^{1}(\Omega)\right\},
\end{aligned}
$$

where $\tilde{H}_{z}$ is a test function and $H^{1}$ is Sobolev space. We note that during the derivation of the weak formulation, the boundary integral for $\Gamma_{\mathrm{PEC}}$ becomes 0 due to the applied PEC condition. Additional details concerning the derivation of the weak formulation for the two-dimensional case are provided in Appendix A.1.

[Fig. 1 about here.]

\subsubsection{Three-dimensional electromagnetic wave propagation problem}

The design domain for the three-dimensional problem is illustrated in Fig.2. Incident waves enter the domain from the left boundary $\Gamma_{1}$. The upper and 
lower boundaries $\Gamma_{\mathrm{PEC}}$ are set as Perfectly Electric Conductors (PEC) and the front and rear boundaries $\Gamma_{\mathrm{PMC}}$ are set as Perfectly Magnetic Conductors (PMC) under periodic conditions. In the three-dimensional case, the following wave propagation equation is derived from Maxwell's equation and the state variable is the electric field $\mathbf{E}$. The relative permeability of both the background material and the dielectric material is again set to 1 .

$$
\nabla \times(\nabla \times \mathbf{E})-k_{0}^{2} \epsilon_{r} \mathbf{E}=0
$$

The boundary conditions are described as follows.

$$
\begin{aligned}
\mathbf{n} \times(\nabla \times \mathbf{E})-j k_{0} \mathbf{n} \times(\mathbf{E} \times \mathbf{n}) & =-2 j k_{0} \mathbf{E}^{i} & & \text { on } \Gamma_{1} \\
\mathbf{n} \times(\nabla \times \mathbf{E})-j k_{0} \mathbf{n} \times(\mathbf{E} \times \mathbf{n}) & =0 & & \text { on } \Gamma_{2} \\
\mathbf{n} \times \mathbf{E} & =0 & & \text { on } \Gamma_{\mathrm{PEC}} \\
\mathbf{n} \times \mathbf{H} & =0 & & \text { on } \Gamma_{\mathrm{PMC}}
\end{aligned}
$$

where $\mathbf{E}^{i}$ is the incident field and $\mathbf{H}$ is the magnetic field. The weak formulation of Eqs.(10)-(14) then derived as follows.

$$
a_{1}(\mathbf{E}, \tilde{\mathbf{E}})+a_{2}(\mathbf{E}, \tilde{\mathbf{E}})=l(\tilde{\mathbf{E}}) \text { for } \quad \mathbf{E} \in U, \quad \tilde{\mathbf{E}} \in U
$$

where

$$
\begin{aligned}
a_{1}(\mathbf{E}, \tilde{\mathbf{E}}) & =\int_{D}(\nabla \times \tilde{\mathbf{E}}) \cdot(\nabla \times \mathbf{E}) d \Omega-k_{0}^{2} \int_{D} \epsilon_{r} \tilde{\mathbf{E}} \cdot \mathbf{E} d \Omega \\
a_{2}(\mathbf{E}, \tilde{\mathbf{E}}) & =j k_{0} \int_{\Gamma_{1} \cup \Gamma_{2}}(\mathbf{n} \times \tilde{\mathbf{E}}) \cdot(\mathbf{n} \times \mathbf{E}) d \Gamma \\
l(\tilde{\mathbf{E}}) & =2 j k_{0} \int_{\Gamma_{1}} \tilde{\mathbf{E}} \cdot \mathbf{E}^{i} d \Gamma \\
U & =\left\{\tilde{\mathbf{E}} \in H^{1}(\Omega)\right\},
\end{aligned}
$$

where $\tilde{\mathbf{E}}$ is a test function. We note that during the derivation of the weak form, the boundary integral for $\Gamma_{\mathrm{PMC}}$ becomes 0 , because $\mathbf{n} \times \mathbf{H}=0$ and the relationship $\nabla \times \mathbf{E}=-j \omega \mu \mathbf{H}$ holds, which is derived from Faraday's law of Maxwell equations, replacing $\partial / \partial t$ with $j \omega$ for time-harmonic electromagnetic fields, and the same is true for $\Gamma_{\mathrm{PEC}}$ when the Galerkin finite element method is used [62]. Additional details for the derivation of the weak formulation for the three-dimensional case are provided in Appendix A.2.

[Fig. 2 about here.] 


\subsection{Effective permeability}

Several approaches can be used to compute an effective property, such as effective permeability and effective permittivity, and these are typically categorized into three types. One approach is to use a homogenization method, such as a method based on the asymptotic expansion $[22,23]$ and the energy-based method [26], another approach is to average the electric and magnetic fields in a unit cell [27], and the third approach is to compute the effective properties based on the S-parameter, namely, the complex transmission and reflection coefficients [28-31]. The first approach can only be applied when the periodic unit cell can be considered as infinitely small compared to the wavelength. Since the unit cell size is relatively large in our design problems, consisting of one unit cell in wave propagation direction, on the order of $1 / 10 \sim 1 / 4$ of the wavelength, this approach is not appropriate. The second approach obtains the effective properties based on the relation of electric field $\mathbf{E}$ and electric flux density $\mathbf{D}$, and also the magnetic field $\mathbf{H}$ and magnetic field density $\mathbf{B}$, using the integral form of Maxwell's equations. However, it has been pointed out that this approach becomes less effective with increasing complexity of metamaterial cell structure [27].

The S-parameter-based approach was first proposed by Smith et al. [28]. Chen et al. [29] proposed an improvement to this method, which can determine the sign of the effective impedance and the correct branch of the real part of the refractive index, but it includes an iterative process, so it is not appropriate as an optimization process because the sensitivity may become exceedingly complicated. In the method of Lubkowski et al. [30], the effective properties are retrieved using parameterized Drude and Lorentz models, but the Drude model does not capture the effective permittivity appropriately for all-dielectric metamaterials. Smith et al. extended their original approach to deal with inhomogeneous cases [31]. Here, we use the extended approach [31], with the effective parameters computed based on S-parameters that can be obtained via the following equations:

$$
\begin{aligned}
S_{11} & =\frac{\int_{\Gamma_{1}}\left(\mathbf{E}-\mathbf{E}^{i}\right) \cdot \mathbf{E}^{i^{*}} d \Gamma}{\int_{\Gamma_{1}} \mathbf{E}^{i} \cdot \mathbf{E}^{i^{*}} d \Gamma} \\
S_{21} & =\frac{\int_{\Gamma_{2}} \mathbf{E} \cdot \mathbf{E}^{i^{*}} d \Gamma}{\int_{\Gamma_{2}} \mathbf{E}^{i} \cdot \mathbf{E}^{i^{*}} d \Gamma} \\
S_{22} & =\frac{\int_{\Gamma_{2}}\left(\mathbf{E}-\mathbf{E}^{i}\right) \cdot \mathbf{E}^{i^{*}} d \Gamma}{\int_{\Gamma_{2}} \mathbf{E}^{i} \cdot \mathbf{E}^{i^{*}} d \Gamma}
\end{aligned}
$$

where $\mathbf{E}^{i *}$ denotes the complex conjugate transpose of $\mathbf{E}^{i}$. The effective per- 
meability $\mu_{\text {eff }}$ is then obtained by following equation.

$$
\mu_{\mathrm{eff}}=Z n,
$$

where

$$
\begin{array}{r}
Z=\sqrt{\frac{\left(1+S_{11}\right)\left(1+S_{22}\right)-S_{21}^{2}}{\left(1-S_{11}\right)\left(1-S_{22}\right)-S_{21}^{2}}} \\
n=\cos ^{-1}\left(\frac{\beta}{2 S_{21}}\right) \frac{\lambda}{2 \pi d}
\end{array}
$$

where $\lambda$ is the wavelength and $d$ is the unit cell length, and

$$
\beta=1+S_{11} S_{22}-S_{21}^{2}
$$

We note that in two-dimensional transverse magnetic (TM) wave propagation problems, $E_{z}=0, H_{x}=0, H_{y}=0$. In addition, $E_{x}$ and $E_{y}$ can be obtained using the following relationship between $\mathbf{E}$ and $\mathbf{H}$, derived from Ampere's law of Maxwell equations, $\nabla \times \mathbf{H}=\partial(\epsilon \mathbf{E}) / \partial t$, and replacing $\partial / \partial t$ with $j \omega$ for time-harmonic electromagnetic fields.

$$
\begin{aligned}
& E_{x}=\frac{1}{j \omega \epsilon} \frac{\partial H_{z}}{\partial y} \\
& E_{y}=\frac{-1}{j \omega \epsilon} \frac{\partial H_{z}}{\partial x}
\end{aligned}
$$

Therefore, $S_{i j}$ can be computed using $H_{z}$ in two-dimensional problems, since $S_{i j}\left(H_{z}\right)$ is a functional of $H_{z}$. We also note that the above formulation [31,44] is an extended formulation of the one provided in an earlier paper [28], where $S_{22}$ is used in addition to $S_{11}$ and $S_{21}$, for inhomogeneous inclusions. By using the extended formulation, symmetric optimized configurations can be obtained because it is symmetric with respect to $S_{11}$ and $S_{22}$.

\subsection{Formulation of optimization problem}

One particularly interesting optimization problem aims to obtain metamaterial designs that exhibit highly negative permeability values. In this case, the objective of the optimization problem is to find a distribution of dielectric material within the fixed design domain that minimizes the effective permeability, and it can be formulated as a problem to minimize the effective permeability at a prescribed frequency. On the other hand, to obtain an effective cloaking device, the metamaterial design must exhibit a certain desirable value for the effective permeability, so the objective of the optimization problem then is to find a distribution of dielectric material that provides the desired value 
of effective permeability, and the optimization problem can be formulated to minimize the square of the difference between the effective permeability and a prescribed value at a prescribed frequency.

\subsubsection{Effective permeability minimization problem}

The purpose of the optimization here is to minimize the real part of the effective permeability at a desired frequency. A typical effective permeability curve is shown in Fig.3(a), where $\mu^{\prime}$ and $\mu^{\prime \prime}$ show the real and imaginary part of the effective permeability, respectively. The real part of the effective permeability has a positive peak as well as an anti-resonance point, where the effective permeability has a desirable negative value. However, if the positive peak lies between the initial anti-resonance point in the optimization and the target frequency, that is, if the target frequency is located in the hatched area for the case shown in Fig.3(a), configurations that demonstrate negative effective permeability cannot be obtained directly because the level set function must always return a lower value of the objective function after updating.

For example, considering a case where the target value is set to $3.0 \mathrm{THz}$ as shown in Fig.3(b), and the real part of the effective permeability is minimized directly, an increase in the frequency of the resonance frequency results in a decrease of the objectives. When the level set function is updated, the antiresonance point moves toward higher frequencies, which prevents obtaining a configuration that demonstrate negative effective permeability. Thus, we use a two-stage optimization procedure [44], where the imaginary part of permeability $\mu^{\prime \prime}$ is minimized during the first stage, taking advantage of the fact that the imaginary part of the permeability does not have positive peak (Fig.3(c)). The optimization problem for this first stage is described as follows.

$$
\begin{array}{ll}
\inf _{\phi} & F=\mu^{\prime \prime} \\
\text { subject to } & G \leq 0 \\
& \text { Governing equation } \\
& \text { Boundary conditions }
\end{array}
$$

where $F$ is the objective functional and $G$ is the constraint functional of the optimization problem. For the second stage of the optimization, the real part of the effective permeability is minimized, using the optimized configuration obtained in the first stage as the initial configuration (Fig.3(d)). The optimization problem for the second stage is described as follows.

$$
\begin{array}{ll}
\inf _{\phi} & F=\mu^{\prime} \\
\text { subject to } & G \leq 0 \\
& \text { Governing equation } \\
& \text { Boundary conditions }
\end{array}
$$


[Fig. 3 about here.]

\subsubsection{Effective permeability design problem}

The purpose of the optimization here is to obtain a distribution of dielectric material which achieves the target value of the effective permeability $\mu_{\mathrm{tar}}^{\prime}$ at a desired frequency. The optimization problem can be formulated as a problem to minimize the square of the difference between the effective permeability and a prescribed value. Again, if the positive peak lies between the initial anti-resonance point in the optimization and the target frequency, obtaining an optimized configuration that demonstrate negative effective permeability directly is problematic, so the two-stage optimization procedure is again used. That is, the imaginary part of the permeability $\mu^{\prime \prime}$ is minimized during the first stage, as described in the previous subsection, and the square of the difference between the effective permeability and a prescribed value is then minimized during the second stage. The optimization problem for the second stage is described as follows.

$$
\begin{array}{ll}
\inf _{\phi} & F=\left(\mu^{\prime}-\mu_{\mathrm{tar}}^{\prime}\right)^{2} \\
\text { subject to } & G \leq 0 \\
& \text { Governing Equation } \\
& \text { Boundary conditions }
\end{array}
$$

\subsection{Level set-based topology optimization method}

Here, we briefly discuss a level set-based topology optimization method that incorporates a fictitious interface energy [60]. A topology optimization problem is formulated using a fixed design domain $D$ that consists of a domain $\Omega$ filled with solid material, a domain filled with void, and structural boundaries $\partial \Omega$. As shown in Fig.4, in the level set-based topology optimization method, the structural boundaries are implicitly represented using the iso-surface of the level set function, as follows.

$$
\left\{\begin{array}{lll}
1 \geq \phi(\mathbf{x})>0 & \text { for } & \forall \mathbf{x} \in \Omega \backslash \partial \Omega \\
\phi(\mathbf{x})=0 & \text { for } & \forall \mathbf{x} \in \partial \Omega \\
0>\phi(\mathbf{x}) \geq-1 & \text { for } & \forall \mathbf{x} \in D \backslash \Omega
\end{array}\right.
$$


[Fig. 4 about here.]

The level set function $\phi$ is used to represent the boundaries of the target structure, where positive values represent the solid domain, negative values represent the void domain, and zero represents the structural boundaries. The optimization problem that minimizes objective functional $F$ under a constraint functional $G$ is then formulated as follows, using the above defined level set function $\phi$.

$$
\begin{aligned}
& \inf _{\chi_{\phi}} \quad F\left(\chi_{\phi}\right)=\int_{D} f_{1}\left(\mathbf{x}, \chi_{\phi}\right) \mathrm{d} \Omega+\int_{\Gamma} f_{2}\left(\mathbf{x}, \chi_{\phi}\right) \mathrm{d} \Gamma \\
& \text { subject to } G\left(\chi_{\phi}\right)=\int_{D} g\left(\mathbf{x}, \chi_{\phi}\right) \mathrm{d} \Omega-G_{\max } \leq 0,
\end{aligned}
$$

where $f_{1}$ and $f_{2}$ are density functions of the objective functional, $g$ is the density function of the constraint functional, and $G_{\max }$ is the upper limit value of $\mathrm{G}$. The characteristic function $\chi_{\phi}(\phi)$ is defined as follows.

$$
\chi_{\phi}(\phi)=\left\{\begin{array}{lll}
1 & \text { if } & \phi \geq 0 \\
0 & \text { if } & \phi<0
\end{array} .\right.
$$

The above optimization problem is an ill-posed problem because the level set function is allowed to be discontinuous at every point, so the optimization problem needs to be regularized. In our method, the Tikhonov regularization method is applied to regularize the optimization problem, and the above formulation is replaced with the following optimization problem:

$$
\begin{aligned}
& \inf _{\phi} F_{R}\left(\chi_{\phi}, \phi\right)=F+R \\
& \text { subject to } G\left(\chi_{\phi}\right) \leq 0,
\end{aligned}
$$

where $R$ in the above equation is defined as follows.

$$
R=\int_{D} \frac{1}{2} \tau|\nabla \phi|^{2} \mathrm{~d} \Omega
$$

In addition, $\tau$ is a regularization parameter that adjusts the degree of regularization. This formulation is then replaced with an optimization problem without constraints, using Lagrange's method of undetermined multipliers, as follows.

$$
\inf _{\phi} \hat{F}_{R}\left(\chi_{\phi}, \phi\right)=\hat{F}+R
$$

where $\hat{F}=F+\lambda G, \hat{F}_{R}$ is the Lagrangian and $\lambda$ is the Lagrange multiplier. Based on the above formulation, the KKT (Karush-Kuhn-Tucker) conditions 
of this optimization problem are described as follows.

$$
\left\langle\frac{d \hat{F}_{R}}{d \phi}, \tilde{\phi}\right\rangle=0, \quad \lambda G=0, \quad \lambda \geq 0, \quad G \leq 0
$$

where the notation $\left\langle\frac{d \hat{F}_{R}}{d \phi}, \tilde{\phi}\right\rangle$ represents the Fréchet derivative of the regularized Lagrangian $\hat{F}_{R}$ with respect to $\phi$.

Level set functions which satisfy the above KKT conditions are candidate solutions of the level set function that represent optimized configurations. However, it is not easy to find optimized solutions directly, so the optimization problem is replaced by a time evolution equation, by introducing a fictitious time $t$. The level set function is updated by solving this equation, and an optimized configuration is ultimately obtained, as explained below.

\subsection{Level set function updating scheme}

For the following formulation, which introduces a fictitious time $t$, we assume that the variation of the level set function is proportional to the gradient of Lagrangian $\hat{F}_{R}$, as follows.

$$
\frac{\partial \phi}{\partial t}=-K(\phi) \frac{d \hat{F}_{R}}{d \phi}
$$

where $K(\phi)>0$ is a coefficient of proportionality. Substituting Eq.(48) into Eq.(50) and setting appropriate boundary conditions, we have the following equations.

$$
\begin{cases}\frac{\partial \phi}{\partial t}=-K(\phi)\left(\frac{d \hat{F}}{d \phi}-\tau \nabla^{2} \phi\right) & \\ \frac{\partial \phi}{\partial n}=0 & \text { on } \partial D \backslash \partial D_{N} \\ \phi=1 & \text { on } \partial D_{N}\end{cases}
$$

The optimized configuration can be then obtained by solving the above time evolution problem. In this research, candidate optimum solutions are found by solving the above equation. Here, since the objective functional $\hat{F}\left(\chi_{\phi}\right)$ is represented as a functional of $\chi_{\phi}$, we note that the derivative of $\hat{F}\left(\chi_{\phi}\right)$ is equivalent to the magnitude of the topological derivative [60,61]. The derivative of $\hat{F}\left(\chi_{\phi}\right)$ can then be obtained as the derivative with respect to $\chi_{\phi}$, namely, $-d \hat{F}\left(\chi_{\phi}\right) / d \chi_{\phi}$. 


\subsection{Sensitivity Analysis}

\subsubsection{Two-dimensional case}

Now, we consider the sensitivity analysis for a two-dimensional case, using the Adjoint Variable Method (AVM). The Lagrangian of the optimization problem is formulated as follows.

$$
\hat{F}=F-\sum_{i j=11,21,22}\left(a_{1}\left(H_{z}, \tilde{H}_{z, i j}\right)+a_{2}\left(H_{z}, \tilde{H}_{z, i j}\right)-l\left(\tilde{H}_{z, i j}\right)\right)+\lambda G,
$$

where $\tilde{H}_{z, i j}$ denotes the adjoint variables with respect to $S_{i j}$. The level set function is updated based on the gradient of the Lagrangian using a reaction diffusion equation. The sensitivity of the Lagrangian is obtained using the AVM, as follows.

$$
\begin{aligned}
&\left\langle\frac{d \hat{F}}{d \chi_{\phi}}, \tilde{\chi}_{\phi}\right\rangle=\sum_{i j=11,21,22}\left\langle\frac{\partial F}{\partial S_{i j}}, \tilde{S}_{i j}\right\rangle\left\langle\frac{\partial S_{i j}}{\partial H_{z}}, \tilde{H}_{z}\right\rangle\left\langle\frac{\partial H_{z}}{\partial \chi_{\phi}}, \tilde{\chi}_{\phi}\right\rangle \\
&-\sum_{i j=11,21,22}\left(\left\langle\frac{\partial a_{1}}{\partial H_{z}}, \tilde{H}_{z}\right\rangle\left\langle\frac{\partial H_{z}}{\partial \chi_{\phi}}, \tilde{\chi}_{\phi}\right\rangle+\left\langle\frac{\partial a_{2}}{\partial H_{z}}, \tilde{H}_{z}\right\rangle\left\langle\frac{\partial H_{z}}{\partial \chi_{\phi}}, \tilde{\chi}_{\phi}\right\rangle\right. \\
&\left.+\left\langle\frac{\partial a_{1}}{\partial \chi_{\phi}}, \tilde{\chi}_{\phi}\right\rangle+\left\langle\frac{\partial a_{2}}{\partial \chi_{\phi}}, \tilde{\chi}_{\phi}\right\rangle-\left\langle\frac{\partial l}{\partial \chi_{\phi}}, \tilde{\chi}_{\phi}\right\rangle\right) \\
&+\lambda\left\langle\frac{\partial G}{\partial \chi_{\phi}}, \tilde{\chi}_{\phi}\right\rangle
\end{aligned}
$$

where $\left\langle\frac{\partial a_{2}}{\partial \chi_{\phi}}, \tilde{\chi}_{\phi}\right\rangle=0$ and $\left\langle\frac{\partial l}{\partial \chi_{\phi}}, \tilde{\chi}_{\phi}\right\rangle=0$, since $a_{2}$ and the incident field are independent of the design variables. Arranging the above equation in order to cancel out the $\left\langle\partial H_{z} / \partial \chi_{\phi}, \tilde{\chi}_{\phi}\right\rangle$ term, the above equation is transformed as follows.

$$
\begin{aligned}
\left\langle\frac{d \hat{F}}{d \chi_{\phi}}, \tilde{\chi}_{\phi}\right\rangle= & \sum_{i j=11,21,22}\left(\left\langle\frac{\partial F}{\partial S_{i j}}, \tilde{S}_{i j}\right\rangle\left\langle\frac{\partial S_{i j}}{\partial H_{z}}, \tilde{H}_{z}\right\rangle\right. \\
& \left.-\left\langle\frac{\partial a_{1}}{\partial H_{z}}, \tilde{H}_{z}\right\rangle-\left\langle\frac{\partial a_{2}}{\partial H_{z}}, \tilde{H}_{z}\right\rangle\right)\left\langle\frac{\partial H_{z}}{\partial \chi_{\phi}}, \tilde{\chi}_{\phi}\right\rangle \\
& -\sum_{i j=11,21,22}\left\langle\frac{\partial a_{1}}{\partial \chi_{\phi}}, \tilde{\chi}_{\phi}\right\rangle
\end{aligned}
$$

where the adjoint variable $\tilde{H}_{z, i j}$ is obtained by solving the following equation.

$$
\begin{array}{r}
a_{1}\left(\tilde{H}_{z, i j}, \delta H_{z}\right)+a_{2}\left(\tilde{H}_{z, i j}, \delta H_{z}\right)=\left\langle\frac{\partial F}{\partial S_{i j}}, \tilde{S}_{i j}\right\rangle\left(S_{i j}\left(\delta H_{z}\right)+\delta_{i j}\right) \\
\quad \text { for } \tilde{H}_{z, i j} \in U,{ }^{\forall} \delta H_{z} \in U,
\end{array}
$$


where $\delta H_{z}$ is the variation of $H_{z}$, and $\delta_{i j}$ is the Kronecker delta such that $\delta_{11}=\delta_{22}=1, \delta_{21}=0$. The sensitivities are finally obtained using the following equation.

$$
\left\langle\frac{d \hat{F}}{d \chi_{\phi}}, \tilde{\chi}_{\phi}\right\rangle=-\sum_{i j=11,21,22}\left\langle\frac{\partial a_{1}\left(H_{z}, \tilde{H}_{z, i j}\right)}{\partial \chi_{\phi}}, \tilde{\chi}_{\phi}\right\rangle+\lambda\left\langle\frac{\partial G}{\partial \chi_{\phi}}, \tilde{\chi}_{\phi}\right\rangle
$$

We note that, although the objective function is separately formulated for the two stages of the optimization, the sensitivities of the real and imaginary parts of the effective permeability are obtained by sensitivity analysis of the complex function. Therefore, the obtained sensitivity $\left\langle\frac{d \hat{F}}{d \chi_{\phi}}, \tilde{\chi}_{\phi}\right\rangle$ is a complex function where the sensitivities of the real and imaginary parts of the objective function are respectively obtained by the real and imaginary parts of the derived sensitivity, namely, $\operatorname{Re}\left(\left\langle\frac{d \hat{F}}{d \chi_{\phi}}, \tilde{\chi}_{\phi}\right\rangle\right)$ and $\operatorname{Im}\left(\left\langle\frac{d \hat{F}}{d \chi_{\phi}}, \tilde{\chi}_{\phi}\right\rangle\right)$. Additional details are provided in Appendix B.

\subsubsection{Three-dimensional case}

The Lagrangian of the optimization problem for a three-dimension case is formulated as follows.

$$
\hat{F}=F-\sum_{i j=11,21,22}\left(a_{1}\left(\mathbf{E}, \tilde{\mathbf{E}}_{i j}\right)+a_{2}\left(\mathbf{E}, \tilde{\mathbf{E}}_{i j}\right)-l\left(\tilde{\mathbf{E}}_{i j}\right)\right)+\lambda G
$$

where $\tilde{\mathbf{E}}_{i j}$ denotes the adjoint variables with respect to $S_{i j}$. The sensitivity of the Lagrangian is obtained as follows.

$$
\begin{gathered}
\left\langle\frac{d \hat{F}}{d \chi_{\phi}}, \tilde{\chi}_{\phi}\right\rangle=\sum_{i j=11,21,22}\left\langle\frac{\partial F}{\partial S_{i j}}, \tilde{S}_{i j}\right\rangle\left\langle\frac{\partial S_{i j}}{\partial \mathbf{E}}, \tilde{\mathbf{E}}\right\rangle \\
-\sum_{i j=11,21,22}\left(\left\langle\frac{\partial a_{1}}{\partial \mathbf{E}}, \tilde{\mathbf{E}}\right\rangle+\left\langle\frac{\partial a_{2}}{\partial \mathbf{E}}, \tilde{\mathbf{E}}\right\rangle+\left\langle\frac{\partial a_{1}}{\partial \chi_{\phi}}, \tilde{\chi}_{\phi}\right\rangle\right)+\lambda\left\langle\frac{\partial G}{\partial \chi_{\phi}}, \tilde{\chi}_{\phi}\right\rangle \\
=\sum_{i j=11,21,22}\left(\left\langle\frac{\partial F}{\partial S_{i j}}, \tilde{S}_{i j}\right\rangle\left\langle\frac{\partial S_{i j}}{\partial \mathbf{E}}, \tilde{\mathbf{E}}\right\rangle-\left\langle\frac{\partial a_{1}}{\partial \mathbf{E}}, \tilde{\mathbf{E}}\right\rangle-\left\langle\frac{\partial a_{2}}{\partial \mathbf{E}}, \tilde{\mathbf{E}}\right\rangle\right) \\
-\sum_{i j=11,21,22}\left\langle\frac{\partial a_{1}}{\partial \chi_{\phi}}, \tilde{\chi}_{\phi}\right\rangle+\lambda\left\langle\frac{\partial G}{\partial \chi_{\phi}}, \tilde{\chi}_{\phi}\right\rangle
\end{gathered}
$$

where the adjoint variable $\tilde{\mathbf{E}}_{i j}$ is obtained by solving the following equation.

$$
\begin{array}{r}
a_{1}\left(\tilde{\mathbf{E}}_{i j}, \delta \mathbf{E}\right)+a_{2}\left(\tilde{\mathbf{E}}_{i j}, \delta \mathbf{E}\right)=\left\langle\frac{\partial F}{\partial S_{i j}}, \tilde{S}_{i j}\right\rangle\left(S_{i j}(\delta \mathbf{E})+\delta_{i j}\right) \\
\text { for } \tilde{\mathbf{E}}_{i j} \in U, \quad{ }^{\forall} \delta \mathbf{E} \in U,
\end{array}
$$


where $\delta \mathbf{E}$ is the variation of $\mathbf{E}$. The sensitivities are then obtained by the following equation.

$$
\left\langle\frac{d \hat{F}}{d \chi_{\phi}}, \tilde{\chi}_{\phi}\right\rangle=-\sum_{i j=11,21,22}\left\langle\frac{\partial a_{1}\left(\tilde{\mathbf{E}}_{i j}, \mathbf{E}\right)}{\partial \chi_{\phi}}, \tilde{\chi}_{\phi}\right\rangle+\lambda\left\langle\frac{\partial G}{\partial \chi_{\phi}}, \tilde{\chi}_{\phi}\right\rangle
$$

We note that in the three-dimensional case, the adjoint equation, Eq.(59), can be solved efficiently, as described below. The left-hand side is the same as that of the governing equation, Eq.(15). Considering the right-hand side, the right-hand side of the adjoint equation is linearly proportional to that of the governing equation for $i j=11$. Therefore, the adjoint field is self-adjoint and its solution is linearly proportional to that of the governing equation. For $i j=21$ and $i j=22$, the solutions of the adjoint equation, Eq.(59), are obtained by switching the location of the input and output boundaries. Further details concerning this approach are given in [34].

\section{$3 \quad$ Numerical implementations}

\subsection{Design variables}

\subsubsection{Two-dimensional case}

The distribution of dielectric material inside the fixed design domain is expressed using the level set function. In our method, we use a reciprocal formulation of the relative electric permittivity to stabilize the optimization calculations, so $\epsilon_{r}$ in the fixed design domain is defined using the characteristic function $\chi_{\phi}$ as follows.

$$
\epsilon_{r}^{-1}=\left(\epsilon_{1}^{-1}-\epsilon_{0}^{-1}\right) \chi_{\phi}(\phi)+\epsilon_{0}^{-1},
$$

where $\epsilon_{1}$ is the relative permittivity of the dielectric material and $\epsilon_{0}$ is the relative permittivity of the background material. For the numerical implementation, the above characteristic function is approximated by the following smoothed Heaviside function $H(\phi)$.

$$
H(\phi)= \begin{cases}0 & (\phi<-w) \\ \frac{1}{2}+\frac{\phi}{w}\left(\frac{15}{16}-\frac{\phi^{2}}{w^{2}}\left(\frac{5}{8}-\frac{3}{16} \frac{\phi^{2}}{w^{2}}\right)\right) & (-w \leq \phi<w) \\ 1 & (w \leq \phi),\end{cases}
$$

where $w$ is the transition width of the Heaviside function, which is set to a sufficiently small value. 
In cases where the relative permittivity is represented using a linear formulation, it is defined as follows.

$$
\epsilon_{r}=\left(\epsilon_{1}-\epsilon_{0}\right) \chi_{\phi}(\phi)+\epsilon_{0}
$$

The $\left\langle\frac{\partial a}{\partial \chi_{\phi}}, \tilde{\chi}_{\phi}\right\rangle$ term used in the sensitivity analysis is given by the following equation.

$$
\left\langle\frac{\partial a}{\partial \chi_{\phi}}, \tilde{\chi}_{\phi}\right\rangle=\int_{D} \frac{-1}{\epsilon_{r}^{2}}\left(\epsilon_{1}-\epsilon_{0}\right) \nabla H_{z} \cdot \nabla \tilde{H}_{z} \tilde{\chi}_{\phi} d \Omega
$$

In the above formulation, the $-1 / \epsilon_{r}^{2}$ term is included in the equation. In level set-based topology optimization methods, structural boundaries are clearly represented by the level set function, so values of the relative permittivity $\epsilon_{r}$ change drastically near these boundaries, assuming values between $\epsilon_{0}$ and $\epsilon_{1}$. Thus, the sensitivity also changes drastically near the structural boundaries, and the sensitivity distribution becomes discontinuous. On the other hand, by using the reciprocal formulation, the $\left\langle\frac{\partial a}{\partial \chi_{\phi}}, \tilde{\chi}_{\phi}\right\rangle$ term used in sensitivity analysis is given by following equation.

$$
\left\langle\frac{\partial a}{\partial \chi_{\phi}}, \tilde{\chi}_{\phi}\right\rangle=\int_{D}\left(\epsilon_{1}^{-1}-\epsilon_{0}^{-1}\right) \nabla H_{z} \cdot \nabla \tilde{H}_{z} \tilde{\chi}_{\phi} d \Omega
$$

In this formulation, $\epsilon_{r}$ is not included in the equation, so the sensitivity distribution remains continuous and optimization calculations are stable. We note that the reciprocal formulation and the linear formulation respectively represent lower and upper theoretical bounds of the effective properties of the composite materials investigated here [63], so the reciprocal formulation is physically reasonable.

\subsubsection{Three-dimensional case}

In the three-dimensional case, the relative electric permittivity $\epsilon_{r}$ is simply defined using the linear formulation in Eq.(63). In this case, the $\left\langle\frac{\partial a}{\partial \chi_{\phi}}, \tilde{\chi}_{\phi}\right\rangle$ term used in the sensitivity analysis is given by the following equation.

$$
\left\langle\frac{\partial a}{\partial \chi_{\phi}}, \tilde{\chi}_{\phi}\right\rangle=-k_{0}^{2} \int_{D}\left(\epsilon_{1}-\epsilon_{0}\right) \tilde{\mathbf{E}} \cdot \mathbf{E} \tilde{\chi}_{\phi} d \Omega
$$

In the above formulation, $\epsilon_{r}$ does not appear as a term in the sensitivity analysis, so the sensitivity distribution remains continuous and optimization calculations are stable. 


\subsection{Optimization algorithm}

\subsubsection{Effective permeability minimization problem}

As described in the previous section, for the problem to minimize the effective permeability, a two-stage optimization procedure is used in which the imaginary part of the effective permeability is first minimized and the real part of the effective permeability is minimized during the second stage.

$1^{\text {st }}$ stage: Minimize imaginary part of effective permeability.

$2^{\text {nd }}$ stage: Minimize real part of effective permeability, using optimized configuration obtained in first stage as initial configuration.

\subsubsection{Effective permeability design problem}

A two-stage optimization procedure is also used in the effective permeability design problem. Assuming that the target permeability is set to a negative value, such as occurs at the anti-resonance point, an optimized configuration that achieves the target value cannot be obtained directly if the positive peak lies between the initial antiresonance point and the target frequency. This behavior is the same as that encountered in the permeability minimization problem. Moreover, in the same manner as in the case when the target value is set to a negative value, when the target permeability is set to a value much larger than 1, such as occurs near the resonance point, an optimized configuration that achieves the target value cannot be obtained directly if the anti-resonance point lies between the initial resonance point and the target frequency. Therefore, we again use a two-stage optimization procedure. The imaginary part of the effective permeability is minimized in the first stage of the optimization, and the square of the difference between the real part of the effective permeability and the effective permeability target value is minimized during the second stage.

$1^{\text {st }}$ stage: Minimize imaginary part of effective permeability.

$2^{\text {nd }}$ stage: Minimize square of difference between real part of effective permeability and target value, using optimized configuration obtained in first stage as initial configuration.

\subsubsection{Flowchart for each stage}

The optimization flowchart for the $1^{\text {st }}$ and $2^{\text {nd }}$ stages described above, are summarized as follows. 
1: Initialize level set function.

2: Solve equilibrium equation using the Finite Element Method (FEM) and calculate the objective functional and constraint functional.

3: If objective functional has converged, terminate the optimization procedure and if not, compute the sensitivities of the objective and constraint functionals using the AVM.

4: Update the level set function using the reaction diffusion equation and return to step 2 of the optimization procedure.

The volume constraint is handled using the augmented Lagrangian method [64] by estimating the Lagrange multiplier $\lambda$ at every iteration to satisfy $G(\phi(t+\Delta t))=0$. Further details are given in [60]. In the following numerical examples, the optimization terminates if the objective function does not improve during 20 consecutive iterations. We use the FEM for solving the equilibrium and adjoint equations for its ease of implementation in the level set-based method we constructed, but other computational methods such as the FDTD method could be applied. We note that the FEM is also stable and fast, especially when applied to single frequency analysis such as in the metamaterial design problems considered here.

\section{Numerical examples}

In this section, several numerical examples are provided to confirm the utility and validity of the presented method for two- and three-dimensional negative permeability dielectric metamaterial design problems.

\subsection{Two-dimensional problems: the effect of positive peak position in the ini- tial configuration}

In the two-dimensional design problems, we first address effective permeability minimization problems where the target frequencies are set either higher or lower than that of the positive peak of the initial configuration, to examine whether the optimization can successfully find optimized configurations that demonstrates negative effective permeability, independent of the location of the positive peak of the initial configuration. Figure 5 shows the design domain and boundary conditions. The size of the analysis domain is set to $120 \mu \mathrm{m} \times$ $120 \mu \mathrm{m}$ and the size of the fixed design domain is set to $80 \mu \mathrm{m} \times 80 \mu \mathrm{m}$. The analysis domain is discretized using $120 \times 120$ square elements. The relative permittivity $\epsilon_{1}$ of the dielectric material is set to $100-1 i$ and the relative permittivity $\epsilon_{0}$ of the background material is set to 1 . The transition width $w$ of the Heaviside function is set to 0.001 . 
[Fig. 5 about here.]

\subsubsection{Effective permeability minimization problem targeting 0.30THz}

For the effective permeability minimization problem, the target frequency is set to $0.30 \mathrm{THz}$ to examine a case where the target frequency is lower than where the anti-resonance point of the initial configuration occurs. A circular rod shape with a volume fraction of $40 \%$ is used as the initial configuration and the upper limit of the volume fraction is set to $70 \%$.

The initial configuration and optimized distribution obtained in the first stage, i.e., the distribution after minimizing the imaginary part of the effective permeability, are shown in Fig. 6. The effective permeability curves for the initial configuration and optimized distribution obtained in the first stage are shown in Fig. 7. The frequency of the negative peak of the imaginary part of the effective permeability gradually decreases during the optimization procedure and finally reaches the prescribed frequency. The value of the imaginary part of the effective permeability of the initial configuration at $0.30 \mathrm{THz}$ is -0.01 , and the frequency of the negative peak of the imaginary part of the effective permeability is approximately $0.41 \mathrm{THz}$. The value of the imaginary part of the effective permeability of the optimized configuration at $0.30 \mathrm{THz}$ is -13.23 . Figure 8 shows the convergence history of the objective function. The objective function sharply decreased after iteration 170 because the negative peak of the imaginary part of the effective permeability approached the target frequency, and the objective function sharply decreases near this peak.

[Fig. 6 about here.]

[Fig. 7 about here.]

[Fig. 8 about here.]

During the second stage of the optimization, the real part of the effective permeability is minimized using the optimized configuration obtained in the first stage as the initial configuration. Figure 9 shows the initial configuration and the optimized configuration obtained in the second stage. The effective permeability curves of the initial and optimized configurations obtained are shown in Fig. 10. The anti-resonance point of the real part of the effective permeability gradually decreases during the optimization procedure and finally reaches the prescribed frequency at the end of the optimization procedure. The values of the real part of the effective permeability of the initial configuration of the first stage and the optimized configuration obtained in the second stage at $0.30 \mathrm{THz}$ are respectively 1.33 and -5.06 , which shows that the optimization successfully found an optimized solution that has negative effective permeability, The values of the imaginary part of the effective permeability of the initial 
configuration used in the first stage of the optimization and the optimized configuration obtained in the second stage at $0.30 \mathrm{THz}$ are respectively -0.01 and -3.22 . The volume fraction of the optimized configuration is $69.8 \%$. Figure 11 shows the convergence history of the objective function during the second stage of the optimization.

Figure 12 shows the magnetic field of the initial configuration for the first stage of the optimization, and that of the optimized configuration obtained after the second stage of the optimization. Note that the ranges in the color bars of the two figures are different. The black arrows in these illustrations indicate the electric field. It can be seen that a circular electric field is generated in the center of the design domain of the optimized configuration, which induces a significant opposing magnetic field along the $z$-axis, whereas the electric field of the initial configuration lacks this feature. Although a comparison of methods for obtaining effective permeabilities is beyond the scope of this paper, we note that the effective permeability can be also obtained as follows, using the method described in $[3,27]$.

$$
\mu_{\mathrm{eff}}=\frac{1}{d} \frac{\int_{D} H_{z}(x, y) d \Omega}{H_{z}(0,0)} .
$$

Here, $H_{z}(0,0)$ is positive, so when $\int_{D} H_{z}(x, y) d \Omega$ is negative, the effective permeability becomes negative. Thus, it is apparent that the strong opposing magnetic field described above is responsible for the obtained negative permeability in the metamaterial.

[Fig. 9 about here.]

[Fig. 10 about here.]

[Fig. 11 about here.]

[Fig. 12 about here.]

\subsubsection{Effective permeability minimization problem targeting $0.45 \mathrm{THz}$}

For the second two-dimensional problem, the target frequency is set to $0.45 \mathrm{THz}$ to examine a case where the target frequency is higher than that of the antiresonance point of the initial configuration. A circular rod shape with a volume fraction of $50 \%$ is used as the initial configuration, but a volume constraint is not applied.

The initial configuration used in the first stage of the optimization and the optimized configuration after the second stage of the optimization are shown in Fig. 13, and the corresponding effective permeability curves for the initial 
and optimized configurations are shown in Fig. 14. The anti-resonance point of the real part of the effective permeability gradually increases during the optimization procedure and finally reaches the prescribed frequency. The real part of the effective permeability of the initial configuration at $0.45 \mathrm{THz}$ is 0.64 , and the frequency at the anti-resonance point of the real part of the effective permeability is approximately $0.37 \mathrm{THz}$. The real part of the effective permeability of the optimized configuration at $0.45 \mathrm{THz}$ is -2.45 , which shows that the optimization successfully found an optimized solution that exhibits negative effective permeability. The values of the imaginary part of the effective permeability of the initial configuration used in the first stage and the optimized configuration obtained in the second stage at $0.45 \mathrm{THz}$ are respectively -0.01 and -2.26 . Figure 15 shows the convergence histories of the objective function during the first and second stages of the optimization, respectively. Figure 16 shows the magnetic field of the initial configuration used in the first stage of the optimization, and that of the optimized configuration obtained after the second stage of the optimization. The black arrows in Fig.16 show the electric field, and we again see that a circular electric field is generated in the center of the design domain of the optimized configuration. A strong opposing magnetic field is also induced in the optimized configuration, which generates the negative permeability.

[Fig. 13 about here.]

[Fig. 14 about here.]

[Fig. 15 about here.]

[Fig. 16 about here.]

\subsection{Effective permeability design problem}

Next, we consider an effective permeability design problem to find an optimized dielectric distribution that exhibits a prescribed value of the effective permeability. The numerical settings of the optimization problem are the same as those used in the previous subsection. The target frequency is set to $0.30 \mathrm{THz}$ and the target value for the effective permeability is set to -3.0. A circular rod shape with a volume fraction of $40 \%$ is used as the initial configuration. The upper limit of the volume fraction is set to $70 \%$. During the first stage of the optimization, the imaginary part of the effective permeability is minimized, as described in subsection 4.1.1. The square of the difference between the effective permeability and a prescribed value is then minimized during the second stage of the optimization.

The initial configuration used in the first stage of the optimization and the 
optimized configuration after the second stage of the optimization are shown in Fig. 17, and the corresponding effective permeability curves for the initial and optimized configurations are shown in Fig. 18. The real part of the effective permeability of the optimized configuration at $0.30 \mathrm{THz}$ is -3.00 , which indicates that the optimization successfully found an optimized configuration that has a desirable value for the effective permeability at the target frequency. The values of the imaginary part of the effective permeability of the initial configuration used in the first stage and the optimized configuration obtained in the second stage at $0.30 \mathrm{THz}$ are respectively -0.01 and -7.44 . Figure 19 shows the convergence history of the objective function during the second stage of the optimization. Figure 20 shows the magnetic field of the initial configuration used in the first stage of the optimization, and that of the optimized configuration obtained after the second stage of the optimization. The black arrows in Fig. 20 show the electric field, and we again see that a strong opposing magnetic field is induced in the center of the design domain of the optimized configuration, which generates the negative permeability.

[Fig. 17 about here.]

[Fig. 18 about here.]

[Fig. 19 about here.]

[Fig. 20 about here.]

\subsection{Two-dimensional problems: high dielectric constant material}

To further verify the usefulness of the present method, we provide effective permeability minimization problems for materials with a high dielectric constant. The numerical settings of the optimization problems are the same as those used in subsection 4.1. The relative permittivity $\epsilon_{1}$ of the dielectric material is set to $200-5 i$ and the relative permittivity $\epsilon_{0}$ of the background material is set to 1. A configuration filled with dielectric material is used as the initial configuration, since we do not consider the effect of the positive peak position here. A volume constraint is not applied in the following examples.

\subsubsection{Effective permeability minimization problem targeting 0.30THz}

Here, the target frequency is set to $0.30 \mathrm{THz}$. The initial configuration used in the first stage of the optimization and the optimized configuration after the second stage of the optimization are shown in Fig. 21, and the corresponding effective permeability curves for the initial and optimized configurations 
are shown in Fig. 22. The anti-resonance point of the real part of the effective permeability gradually increases during the optimization procedure and finally reaches the prescribed frequency. The real part of the effective permeability of the initial configuration at $0.30 \mathrm{THz}$ is 0.70 , and the frequency at the anti-resonance point of the real part of the effective permeability is approximately $0.19 \mathrm{THz}$. The real part of the effective permeability of the optimized configuration at $0.30 \mathrm{THz}$ is -2.89 , which shows that the optimization successfully found an optimized solution that exhibits negative effective permeability. The values of the imaginary part of the effective permeability of the initial configuration used in the first stage and the optimized configuration obtained in the second stage at $0.30 \mathrm{THz}$ are respectively 0.00 and -2.26 . Figure 23 shows the convergence histories of the objective function during the first and second stages of the optimization. Figure 24 shows the magnetic field of the initial configuration used in the first stage of the optimization, and that of the optimized configuration obtained after the second stage of the optimization. Once more, a strong opposing magnetic field is induced in the left and right-center areas of the optimized configuration, which generates the negative permeability.

[Fig. 21 about here.]

[Fig. 22 about here.]

[Fig. 23 about here.]

[Fig. 24 about here.]

\subsubsection{Effective permeability minimization problem targeting $0.45 \mathrm{THz}$}

Next, the target frequency is set to $0.45 \mathrm{THz}$. The initial configuration used in the first stage of the optimization and the optimized configuration after the second stage of the optimization are shown in Fig. 25, and the corresponding effective permeability curves for the initial and optimized configurations are shown in Fig. 26. Again, the anti-resonance point of the real part of the effective permeability gradually increases during the optimization procedure and finally reaches the prescribed frequency. The real part of the effective permeability of the initial configuration at $0.45 \mathrm{THz}$ is 0.22 , and the frequency at the anti-resonance point of the real part of the effective permeability is approximately $0.43 \mathrm{THz}$. The real part of the effective permeability of the optimized configuration at $0.45 \mathrm{THz}$ is -0.58 , which shows that the optimization successfully found an optimized solution that exhibits negative effective permeability. The values of the imaginary part of the effective permeability of the initial configuration used in the first stage and the optimized configuration obtained in the second stage at $0.45 \mathrm{THz}$ are respectively -0.08 and -1.18 . Figure 27 shows the convergence histories of the objective function during the 
first and second stages of the optimization. Figure 28 shows the magnetic field of the initial configuration used in the first stage of the optimization, and the optimized configuration obtained after the second stage of the optimization. Although a strong magnetic field is induced in the center of the optimized configuration, a strong opposing magnetic field is induced in areas around the center, generating a negative permeability overall.

[Fig. 25 about here.]

[Fig. 26 about here.]

[Fig. 27 about here.]

[Fig. 28 about here.]

\subsection{Three-dimensional problems}

We now consider two three-dimensional effective permeability minimization problems where the target frequencies are either higher or lower than that of the positive peak of the initial configuration, to show that the optimization can successfully find optimized configurations that demonstrates negative effective permeability, regardless of the location of the positive peak of the initial configuration. Figure 29 shows the design domain and boundary conditions. The size of the analysis domain is set to $120 \mu \mathrm{m} \times 120 \mu \mathrm{m} \times 150 \mu \mathrm{m}$ and the size of the fixed design domain is set to $80 \mu \mathrm{m} \times 80 \mu \mathrm{m} \times 110 \mu \mathrm{m}$. The analysis domain is discretized using $48 \times 48 \times 60$ square elements. The relative permittivity $\epsilon_{1}$ of the dielectric material is set to $100-1 i$ and the relative permittivity $\epsilon_{0}$ of the background material is set to 1 . The transition width $w$ of the Heaviside function is set to 0.001 .

[Fig. 29 about here.]

\subsubsection{Effective permeability minimization problem targeting 0.30THz}

The effective permeability minimization problem where the target frequency is set to $0.30 \mathrm{THz}$ examines a case where the target frequency is lower than that of the anti-resonance point of the initial configuration. A spherical shape with a volume fraction of $25 \%$ is used as the initial configuration. The upper limit of the volume fraction is set to $90 \%$. The initial configuration used in the first stage of the optimization and the optimized configuration after the second stage of the optimization are shown in Fig. 30, and the corresponding 
effective permeability curves are shown in Fig. 31. The anti-resonance point of the real part of the effective permeability gradually decreases during the optimization procedure and finally reaches the prescribed frequency. The real part of the effective permeability of the initial and optimized configurations at $0.30 \mathrm{THz}$ are respectively 1.06 and -3.48 , which shows that the optimization can successfully find an optimized solution that demonstrates negative effective permeability. The values of the imaginary part of the effective permeability used in the initial configuration of the first stage and the optimized configuration obtained in the second stage at $0.30 \mathrm{THz}$ are respectively 0.00 and -2.68 . The volume fraction of the optimized configuration is $90.0 \%$. Figure 32 shows the convergence histories of the objective function during the first and second stages of the optimization.

[Fig. 30 about here.]

[Fig. 31 about here.]

[Fig. 32 about here.]

\subsubsection{Effective permeability minimization problem targeting $0.45 \mathrm{THz}$}

The effective permeability minimization problem where the target frequency is set to $0.45 \mathrm{THz}$ now examines a case where the target frequency is higher than that of the anti-resonance point of the initial configuration. A spherical shape with a volume fraction of $40 \%$ is used as the initial configuration, and a volume constraint is not applied. The initial configuration used in the first stage of the optimization and the optimized configuration after the second stage of the optimization are shown in Fig. 33, and the corresponding effective permeability curves are shown in Fig. 34. The anti-resonance point of the real part of the effective permeability gradually increases during the optimization procedure and finally reaches the prescribed frequency. The real part of the effective permeability of the initial and optimized configuration at $0.45 \mathrm{THz}$ are respectively 0.64 and -1.61 , which shows that the optimization can successfully find an optimized solution that has negative effective permeability. The values of the imaginary part of the effective permeability of the initial configuration used in the first stage and the optimized configuration obtained in the second stage at $0.45 \mathrm{THz}$ are respectively -0.01 and -1.99 . Figure 35 shows the convergence histories of the objective function during the first and second stages.

[Fig. 33 about here.]

[Fig. 34 about here.]

[Fig. 35 about here.] 


\section{Conclusions}

This paper presented a level set-based topology optimization method for the design of negative permeability dielectric metamaterials. We achieved the following:

(1) The optimization problems for both two- and three-dimensional problems were formulated to minimize the effective permeability, and to obtain a prescribed effective permeability at a target frequency. A level set-based boundary expression was applied to obtain clear boundaries, and an S-parameter-based approach was applied to compute the effective permeability of the metamaterials.

(2) Based on the formulation of the optimization problem, an optimization algorithm was constructed. The FEM was used to solve the electromagnetic wave problems and update the level set function, and the Adjoint Variable Method was used when computing sensitivity analyses.

(3) Several numerical examples for both two- and three-dimensional problems were provided to examine the validity of the presented method. We can confirm that our method successfully finds optimized configurations that minimize the effective permeability, and also finds optimized configurations that achieve a prescribed degree of effective permeability. Finally, we also confirm that the presented method obtains smooth and clear optimized configurations for all the presented cases.

\section{Acknowledgments}

This work is supported by JSPS Grant-in-Aid for Scientific Research (B), 22360041, and JSPS "Institutional Program for Young Researcher Overseas Visits." The first author is partially supported by Aisin AW Co., Ltd. We sincerely appreciate all of these forms of assistance. 


\section{A Derivation of weak formulation}

\section{A.1 Two-dimensional case}

Here, the weak formulation of the governing equation is derived for the twodimensional case. Multiplying Eq.(1) by a test function $\tilde{H}_{z}$, and integrating over domain $D$, we have,

$$
\int_{D} \tilde{H}_{z}\left[\nabla \cdot\left(\epsilon_{r}^{-1} \nabla H_{z}\right)\right] d \Omega+\int_{D} \tilde{H}_{z}\left(k_{0}^{2} H_{z}\right) d \Omega=0 .
$$

The first term on the left-hand side of above equation can be transformed as follows, applying the method of integration by parts.

$$
\int_{D} \tilde{H}_{z}\left[\nabla \cdot\left(\epsilon_{r}^{-1} \nabla H_{z}\right)\right] d \Omega=\int_{D} \nabla \cdot\left(\tilde{H}_{z} \epsilon_{r}^{-1} \nabla H_{z}\right) d \Omega-\int_{D} \nabla \tilde{H}_{z} \cdot\left(\epsilon_{r}^{-1} \nabla H_{z}\right) d \Omega .
$$

Moreover, the first term on the right-hand side of the above equation can be expressed by a boundary integral, using Gauss's theorem, as follows.

$$
\int_{D} \nabla \cdot\left(\tilde{H}_{z} \epsilon_{r}^{-1} \nabla H_{z}\right) d \Omega=\int_{\Gamma} \tilde{H}_{z}\left[\mathbf{n} \cdot\left(\epsilon_{r}^{-1} \nabla H_{z}\right)\right] d \Gamma .
$$

Substituting the above two equations into Eq.(A.1), we obtain,

$$
\int_{\Gamma} \tilde{H}_{z}\left[\mathbf{n} \cdot\left(\epsilon_{r}^{-1} \nabla H_{z}\right)\right] d \Gamma-\int_{D} \nabla \tilde{H}_{z} \cdot\left(\epsilon_{r}^{-1} \nabla H_{z}\right) d \Omega+k_{0}^{2} \int_{D} \tilde{H}_{z} H_{z} d \Omega=0 .
$$

Substituting the boundary conditions expressed in Eqs.(2)-(4) into the boundary integral of the above equation, we obtain the following weak formulation.

$\int_{D} \nabla \tilde{H}_{z} \cdot\left(\epsilon_{r}^{-1} \nabla H_{z}\right) d \Omega-k_{0}^{2} \int_{D} \tilde{H}_{z} H_{z} d \Omega+j k_{0} \int_{\Gamma_{1} \cup \Gamma_{2}} \tilde{H}_{z} H_{z} d \Gamma=2 j k_{0} \int_{\Gamma_{1}} H_{z}^{i} \tilde{H}_{z} d \Gamma$

where the boundary integral for $\Gamma_{\mathrm{PEC}}$ in Eq.(A.4) assumes a value of 0 due to the PEC condition, $\mathbf{n} \cdot\left(\epsilon_{r}^{-1} \nabla H_{z}\right)=0$.

\section{A.2 Three-dimensional case}

Next, the weak formulation of the governing equation is derived for the threedimensional case. Multiplying Eq.(10) by a test function $\tilde{\mathbf{E}}$, and integrating over domain $D$, we have,

$$
\int_{D} \tilde{\mathbf{E}} \cdot[\nabla \times(\nabla \times \mathbf{E})] d \Omega-\int_{D} \tilde{\mathbf{E}} \cdot\left(k_{0}^{2} \epsilon_{r} \mathbf{E}\right) d \Omega=0 .
$$


Invoking the following vector identity,

$$
\tilde{\mathbf{E}} \cdot[\nabla \times(\nabla \times \mathbf{E})]=(\nabla \times \tilde{\mathbf{E}}) \cdot(\nabla \times \mathbf{E})-\nabla \cdot[\tilde{\mathbf{E}} \times(\nabla \times \mathbf{E})],
$$

and using Gauss's theorem, we obtain,

$$
\begin{aligned}
\int_{D} \nabla \cdot[\tilde{\mathbf{E}} \times(\nabla \times \mathbf{E})] d \Omega & =\int_{\Gamma} \mathbf{n} \cdot[\tilde{\mathbf{E}} \times(\nabla \times \mathbf{E})] d \Gamma \\
& =-\int_{\Gamma} \tilde{\mathbf{E}} \cdot[\mathbf{n} \times(\nabla \times \mathbf{E})] d \Gamma
\end{aligned}
$$

where the vector identity $\mathbf{a} \cdot(\mathbf{b} \times \mathbf{c})=\mathbf{b} \cdot(\mathbf{c} \times \mathbf{a})$ is applied in the second line of the above equation. Substituting the above two equations into Eq.(A.6), we obtain,

$$
\int_{D}(\nabla \times \tilde{\mathbf{E}}) \cdot(\nabla \times \mathbf{E}) d \Omega+\int_{\Gamma} \tilde{\mathbf{E}} \cdot[\mathbf{n} \times(\nabla \times \mathbf{E})] d \Gamma-\int_{D} \tilde{\mathbf{E}} \cdot\left(k_{0}^{2} \epsilon_{r} \mathbf{E}\right) d \Omega=0 .
$$

Substituting the boundary conditions described in Eqs.(11)-(14) into the boundary integral of the above equation,

$$
\begin{array}{r}
\int_{D}(\nabla \times \tilde{\mathbf{E}}) \cdot(\nabla \times \mathbf{E}) d \Omega-k_{0}^{2} \int_{D} \epsilon_{r} \tilde{\mathbf{E}} \cdot \mathbf{E} d \Omega+j k_{0} \int_{\Gamma_{1} \cup \Gamma_{2}} \tilde{\mathbf{E}} \cdot[\mathbf{n} \times(\mathbf{E} \times \mathbf{n})] d \Gamma \\
=2 j k_{0} \int_{\Gamma_{1}} \tilde{\mathbf{E}} \cdot \mathbf{E}^{i} d \Gamma . \quad(\mathrm{A} .11)
\end{array}
$$

Applying the vector identities $\mathbf{a} \cdot(\mathbf{b} \times \mathbf{c})=\mathbf{b} \cdot(\mathbf{c} \times \mathbf{a})$ and $(\mathbf{a} \times \mathbf{b})=-(\mathbf{b} \times \mathbf{a})$ for the third term on the left-hand side of the above equation, we obtain the following weak formulation.

$$
\begin{array}{r}
\int_{D}(\nabla \times \tilde{\mathbf{E}}) \cdot(\nabla \times \mathbf{E}) d \Omega-k_{0}^{2} \int_{D} \epsilon_{r} \tilde{\mathbf{E}} \cdot \mathbf{E} d \Omega+j k_{0} \int_{\Gamma_{1} \cup \Gamma_{2}}(\mathbf{n} \times \tilde{\mathbf{E}}) \cdot(\mathbf{n} \times \mathbf{E}) d \Gamma \\
=2 j k_{0} \int_{\Gamma_{1}} \tilde{\mathbf{E}} \cdot \mathbf{E}^{i} d \Gamma \quad(\mathrm{A} .12)
\end{array}
$$

We note that the boundary integral in Eq.(A.10) for $\Gamma_{\mathrm{PMC}}$ assumes a value of 0 because $\mathbf{n} \times \mathbf{H}=0$. That is, $\mathbf{n} \times(\nabla \times \mathbf{E})=0$, based on the relationship $\nabla \times \mathbf{E}=$ $-j \omega \mu \mathbf{H}$, which is derived from Faraday's law of Maxwell equations, $\nabla \times \mathbf{E}=$ $-\partial(\mu \mathbf{H}) / \partial t$, replacing $\partial / \partial t$ with $j \omega$ for time-harmonic electromagnetic fields. We also note that the same is true for $\Gamma_{\mathrm{PEC}}$ when the Galerkin finite element method is used, where $\mathbf{n} \times \tilde{\mathbf{E}}=0$ holds on $\Gamma_{\text {PEC }}$. The integrand of the boundary integral in Eq.(A.10) can be transformed as $\tilde{\mathbf{E}} \cdot[\mathbf{n} \times(\nabla \times \mathbf{E})]=(\mathbf{n} \times \tilde{\mathbf{E}})$. $(\nabla \times \mathbf{E})$, applying the vector identity. Thus, the boundary integral for $\Gamma_{\mathrm{PEC}}$ assumes a value of 0 [62]. 


\section{B Sensitivity analysis of a complex function using Adjoint Variable Method}

In subsection 2.6, the sensitivities for the real and the imaginary parts of the objective function are derived simultaneously using the AVM. In this appendix, we show that these sensitivities are equivalent to the sensitivities of the real and imaginary parts obtained individually using the AVM. Although we use the strong form here to clarify the derivation, the equivalence of the sensitivities would be the same for a derivation using the weak form.

Let $\Psi$ be the objective function that has complex values, namely, $\Psi=\Psi^{\prime}+i \Psi^{\prime \prime}$. The sensitivities for the real and imaginary parts can be simultaneously obtained following the standard AVM in the following procedure. First, an additional term is added to the original objective function $\Psi$, as follows.

$$
\hat{\Psi}=\Psi(\phi, \mathbf{u})+\lambda^{T}(\mathbf{S u}-\mathbf{f}),
$$

where $\phi$ is the design variables, $\lambda$ is the adjoint variables, $\mathbf{S}$ is the stiffness matrix, and $\mathbf{f}$ is the load vector. The sensitivity of the objective function is then given as,

$$
\begin{aligned}
\frac{d \hat{\Psi}}{d \phi} & =\frac{\partial \Psi}{\partial \phi}+\frac{\partial \Psi}{\partial \mathbf{u}} \frac{\partial \mathbf{u}}{\partial \phi}+\lambda^{T}\left(\frac{\partial \mathbf{S}}{\partial \phi} \mathbf{u}+\mathbf{S} \frac{\partial \mathbf{u}}{\partial \phi}-\frac{\partial \mathbf{f}}{\partial \phi}\right) \\
& =\frac{\partial \Psi}{\partial \phi}+\lambda^{T}\left(\frac{\partial \mathbf{S}}{\partial \phi} \mathbf{u}-\frac{\partial \mathbf{f}}{\partial \phi}\right)+\left(\frac{\partial \Psi}{\partial \mathbf{u}}+\lambda^{T} \mathbf{S}\right) \frac{\partial \mathbf{u}}{\partial \phi}
\end{aligned}
$$

The sensitivity can then be written as,

$$
\frac{d \hat{\Psi}}{d \phi}=\frac{\partial \Psi}{\partial \phi}+\lambda^{T}\left(\frac{\partial \mathbf{S}}{\partial \phi} \mathbf{u}-\frac{\partial \mathbf{f}}{\partial \phi}\right),
$$

where the adjoint variable $\lambda$ satisfies the following adjoint equation.

$$
\mathbf{S} \lambda=-\left(\frac{\partial \Psi}{\partial \mathbf{u}}\right)^{T} .
$$

The sensitivities of the real and imaginary parts of the objective function can be obtained by the real and imaginary parts of the obtained sensitivity, respectively.

Next, we consider the sensitivities for the real and imaginary parts of the objective function $\Psi$ individually, and show that the obtained sensitivities are the same as the real and imaginary parts of the sensitivity obtained by Eq.(B.3). First, we derive the sensitivity for the real part of the objective function $\Psi^{\prime}$. Following the AVM, two extra terms are added to the original objective function in order to cancel out the imaginary part, as follows $[65,66]$, in the case 
where the response of the governing equation $u$ is a complex function, namely $u=u^{\prime}+i u^{\prime \prime}$.

$$
\hat{\Psi}^{\prime}=\Psi^{\prime}\left(\phi, \mathbf{u}^{\prime}, \mathbf{u}^{\prime \prime}\right)+\lambda_{R}^{T}(\mathbf{S u}-\mathbf{f})+\bar{\lambda}_{R}^{T}(\overline{\mathbf{S}} \overline{\mathbf{u}}-\overline{\mathbf{f}})
$$

where $\lambda_{R}$ is the adjoint variable and subscript $R$ indicates the correspondence to the real part of objective function. $\bar{\lambda}_{R}$ denotes the complex conjugate of $\lambda_{R}$. The sensitivity of the objective function is then given as,

$$
\begin{aligned}
\frac{d \hat{\Psi}^{\prime}}{d \phi} & =\frac{\partial \Psi^{\prime}}{\partial \phi}+\frac{\partial \Psi^{\prime}}{\partial \mathbf{u}^{\prime}} \frac{\partial \mathbf{u}^{\prime}}{\partial \phi}+\frac{\partial \Psi^{\prime}}{\partial \mathbf{u}^{\prime \prime}} \frac{\partial \mathbf{u}^{\prime \prime}}{\partial \phi} \\
& +\lambda_{R}^{T}\left(\frac{\partial \mathbf{S}}{\partial \phi} \mathbf{u}^{\prime}+\mathbf{S} \frac{\partial \mathbf{u}^{\prime}}{\partial \phi}+i \frac{\partial \mathbf{S}}{\partial \phi} \mathbf{u}^{\prime \prime}+i \mathbf{S} \frac{\partial \mathbf{u}^{\prime \prime}}{\partial \phi}-\frac{\partial \mathbf{f}}{\partial \phi}\right) \\
& +\bar{\lambda}_{R}^{T}\left(\frac{\partial \overline{\mathbf{S}}}{\partial \phi} \mathbf{u}^{\prime}+\overline{\mathbf{S}} \frac{\partial \mathbf{u}^{\prime}}{\partial \phi}-i \frac{\partial \overline{\mathbf{S}}}{\partial \phi} \mathbf{u}^{\prime \prime}-i \overline{\mathbf{S}} \frac{\partial \mathbf{u}^{\prime \prime}}{\partial \phi}-\frac{\partial \overline{\mathbf{f}}}{\partial \phi}\right)
\end{aligned}
$$

Rearranging the above equation, we obtain following.

$$
\begin{aligned}
\frac{d \hat{\Psi}^{\prime}}{d \phi} & =\frac{\partial \Psi^{\prime}}{\partial \phi}+\lambda_{R}^{T}\left(\frac{\partial \mathbf{S}}{\partial \phi} \mathbf{u}^{\prime}+i \frac{\partial \mathbf{S}}{\partial \phi} \mathbf{u}^{\prime \prime}-\frac{\partial \mathbf{f}}{\partial \phi}\right)+\bar{\lambda}_{R}^{T}\left(\frac{\partial \overline{\mathbf{S}}}{\partial \phi} \mathbf{u}^{\prime}-i \frac{\partial \overline{\mathbf{S}}}{\partial \phi} \mathbf{u}^{\prime \prime}-\frac{\partial \overline{\mathbf{f}}}{\partial \phi}\right) \\
& +\left(\frac{\partial \Psi^{\prime}}{\partial \mathbf{u}^{\prime}}+\lambda_{R}^{T} \mathbf{S}+\bar{\lambda}_{R}^{T} \overline{\mathbf{S}}\right) \frac{\partial \mathbf{u}^{\prime}}{\partial \phi}+\left(\frac{\partial \Psi^{\prime}}{\partial \mathbf{u}^{\prime \prime}}+i \lambda_{R}^{T} \mathbf{S}-i \bar{\lambda}_{R}^{T} \overline{\mathbf{S}}\right) \frac{\partial \mathbf{u}^{\prime \prime}}{\partial \phi}
\end{aligned}
$$

To delete the $\frac{\partial \mathbf{u}^{\prime}}{\partial \phi}$ and $\frac{\partial \mathbf{u}^{\prime \prime}}{\partial \phi}$ terms, the following two equations must be satisfied.

$$
\begin{array}{r}
\lambda_{R}^{T} \mathbf{S}+\bar{\lambda}_{R}^{T} \overline{\mathbf{S}}=-\frac{\partial \Psi^{\prime}}{\partial \mathbf{u}^{\prime}} \\
i \lambda_{R}^{T} \mathbf{S}-i \bar{\lambda}_{R}^{T} \overline{\mathbf{S}}=-\frac{\partial \Psi^{\prime}}{\partial \mathbf{u}^{\prime \prime}}
\end{array}
$$

From the above equations, the following two adjoint equations are obtained.

$$
\begin{aligned}
& \mathbf{S} \lambda_{R}=-\frac{1}{2}\left(\frac{\partial \Psi^{\prime}}{\partial \mathbf{u}^{\prime}}-i \frac{\partial \Psi^{\prime}}{\partial \mathbf{u}^{\prime \prime}}\right)^{T} \\
& \overline{\mathbf{S}} \bar{\lambda}_{R}=-\frac{1}{2}\left(\frac{\partial \Psi^{\prime}}{\partial \mathbf{u}^{\prime}}+i \frac{\partial \Psi^{\prime}}{\partial \mathbf{u}^{\prime \prime}}\right)^{T}
\end{aligned}
$$

The above two adjoint equations are equivalent, so $\lambda_{R}$ can be obtained by solving Eq.(B.10). Finally, the sensitivity for the real part is obtained as follows.

$$
\frac{d \hat{\Psi}^{\prime}}{d \phi}=\frac{\partial \Psi^{\prime}}{\partial \phi}+\lambda_{R}^{T}\left(\frac{\partial \mathbf{S}}{\partial \phi} \mathbf{u}-\frac{\partial \mathbf{f}}{\partial \phi}\right)+\bar{\lambda}_{R}^{T}\left(\frac{\partial \overline{\mathbf{S}}}{\partial \phi} \overline{\mathbf{u}}-\frac{\partial \overline{\mathbf{f}}}{\partial \phi}\right)
$$


where

$$
\mathbf{S} \lambda_{R}=-\frac{1}{2}\left(\frac{\partial \Psi^{\prime}}{\partial \mathbf{u}^{\prime}}-i \frac{\partial \Psi^{\prime}}{\partial \mathbf{u}^{\prime \prime}}\right)^{T}
$$

Following the same procedure, the sensitivity for the imaginary part of the objective function is obtained as follows.

$$
\frac{d \hat{\Psi}^{\prime \prime}}{d \phi}=\frac{\partial \Psi^{\prime \prime}}{\partial \phi}+\lambda_{I}^{T}\left(\frac{\partial \mathbf{S}}{\partial \phi} \mathbf{u}-\frac{\partial \mathbf{f}}{\partial \phi}\right)+\bar{\lambda}_{I}^{T}\left(\frac{\partial \overline{\mathbf{S}}}{\partial \phi} \overline{\mathbf{u}}-\frac{\partial \overline{\mathbf{f}}}{\partial \phi}\right)
$$

where $\lambda_{I}$ is the adjoint variable that corresponds to the imaginary part of the objective function, and

$$
\mathbf{S} \lambda_{I}=-\frac{1}{2}\left(\frac{\partial \Psi^{\prime \prime}}{\partial \mathbf{u}^{\prime}}-i \frac{\partial \Psi^{\prime \prime}}{\partial \mathbf{u}^{\prime \prime}}\right)^{T}
$$

Multiplying Eq.(B.14) by $i$ and adding the result to Eq.(B.12), we obtain

$$
\begin{aligned}
\frac{d \hat{\Psi}^{\prime}}{d \phi}+i \frac{d \hat{\Psi}^{\prime \prime}}{d \phi} & =\frac{\partial \Psi^{\prime}}{\partial \phi}+i \frac{\partial \Psi^{\prime \prime}}{\partial \phi} \\
& +\left(\lambda_{R}+i \lambda_{I}\right)^{T}\left(\frac{\partial \mathbf{S}}{\partial \phi} \mathbf{u}-\frac{\partial \mathbf{f}}{\partial \phi}\right)+\left(\bar{\lambda}_{R}+i \bar{\lambda}_{I}\right)^{T}\left(\frac{\partial \overline{\mathbf{S}}}{\partial \phi} \overline{\mathbf{u}}-\frac{\partial \overline{\mathbf{f}}}{\partial \phi}\right)
\end{aligned}
$$

Considering the conjugate form of Eq.(B.13) and Eq.(B.15),

$$
\begin{aligned}
& \overline{\mathbf{S}} \bar{\lambda}_{R}=-\frac{1}{2}\left(\frac{\partial \Psi^{\prime}}{\partial \mathbf{u}^{\prime}}+i \frac{\partial \Psi^{\prime}}{\partial \mathbf{u}^{\prime \prime}}\right)^{T} \\
& \overline{\mathbf{S}} \bar{\lambda}_{I}=-\frac{1}{2}\left(\frac{\partial \Psi^{\prime \prime}}{\partial \mathbf{u}^{\prime}}+i \frac{\partial \Psi^{\prime \prime}}{\partial \mathbf{u}^{\prime \prime}}\right)^{T}
\end{aligned}
$$

Multiplying Eq.(B.18) by $i$ and adding the result to Eq.(B.17), we obtain the following.

$$
\begin{aligned}
\overline{\mathbf{S}}\left(\bar{\lambda}_{R}+i \bar{\lambda}_{I}\right) & =-\frac{1}{2}\left(\left(\frac{\partial \Psi^{\prime}}{\partial \mathbf{u}^{\prime}}+i \frac{\partial \Psi^{\prime}}{\partial \mathbf{u}^{\prime \prime}}\right)+i\left(\frac{\partial \Psi^{\prime \prime}}{\partial \mathbf{u}^{\prime}}+i \frac{\partial \Psi^{\prime \prime}}{\partial \mathbf{u}^{\prime \prime}}\right)\right)^{T} \\
& =-\frac{1}{2}\left(\left(\frac{\partial \Psi^{\prime}}{\partial \mathbf{u}^{\prime}}+i \frac{\partial \Psi^{\prime \prime}}{\partial \mathbf{u}^{\prime}}\right)+i\left(\frac{\partial \Psi^{\prime}}{\partial \mathbf{u}^{\prime \prime}}+i \frac{\partial \Psi^{\prime \prime}}{\partial \mathbf{u}^{\prime \prime}}\right)\right)^{T} \\
& =-\frac{1}{2}\left(\frac{\partial \Psi}{\partial \mathbf{u}^{\prime}}+i \frac{\partial \Psi}{\partial \mathbf{u}^{\prime \prime}}\right)^{T}
\end{aligned}
$$


When $\Psi$ can be expressed explicitly using $\mathbf{u}$ (without using $\mathbf{u}^{\prime}$ and/or $\mathbf{u}^{\prime \prime}$ ), the following relationships hold.

$$
\begin{gathered}
\frac{\partial \Psi}{\partial \mathbf{u}^{\prime}}=\frac{\partial \Psi}{\partial \mathbf{u}} \frac{\partial \mathbf{u}}{\partial \mathbf{u}^{\prime}}=\frac{\partial \Psi}{\partial \mathbf{u}} \\
\frac{\partial \Psi}{\partial \mathbf{u}^{\prime \prime}}=\frac{\partial \Psi}{\partial \mathbf{u}} \frac{\partial \mathbf{u}}{\partial \mathbf{u}^{\prime \prime}}=i \frac{\partial \Psi}{\partial \mathbf{u}}
\end{gathered}
$$

Substituting the above equations into Eq.(B.19),

$$
\overline{\mathbf{S}}\left(\bar{\lambda}_{R}+i \bar{\lambda}_{I}\right)=-\frac{1}{2}\left(\frac{\partial \Psi}{\partial \mathbf{u}}-\frac{\partial \Psi}{\partial \mathbf{u}}\right)^{T}=0 .
$$

Therefore, $\bar{\lambda}_{R}+i \bar{\lambda}_{I}=0$, because the stiffness matrix $\mathbf{S}$ is a full-rank matrix. Substituting this into Eq.(B.16), we obtain,

$$
\frac{d \hat{\Psi}}{d \phi}=\frac{\partial \Psi}{\partial \phi}+\lambda^{T}\left(\frac{\partial \mathbf{S}}{\partial \phi} \mathbf{u}-\frac{\partial \mathbf{f}}{\partial \phi}\right),
$$

where $\lambda=\lambda_{R}+i \lambda_{I}$. Eq.(B.23) is equivalent to Eq.(B.3). In addition, the adjoint variable $\lambda$ is obtained as follows. Multiplying Eq.(B.13) by $i$ and adding the result to Eq.(B.15), we have,

$$
\begin{aligned}
\mathbf{S}\left(\lambda_{R}+i \lambda_{I}\right) & =-\frac{1}{2}\left(\left(\frac{\partial \Psi^{\prime}}{\partial \mathbf{u}^{\prime}}-i \frac{\partial \Psi^{\prime}}{\partial \mathbf{u}^{\prime \prime}}\right)+i\left(\frac{\partial \Psi^{\prime \prime}}{\partial \mathbf{u}^{\prime}}-i \frac{\partial \Psi^{\prime \prime}}{\partial \mathbf{u}^{\prime \prime}}\right)\right)^{T} \\
& =-\frac{1}{2}\left(\frac{\partial \Psi}{\partial \mathbf{u}^{\prime}}-i \frac{\partial \Psi}{\partial \mathbf{u}^{\prime \prime}}\right)^{T}
\end{aligned}
$$

Using the same relationships for Eqs.(B.20) and (B.21),

$$
\mathbf{S} \lambda=-\left(\frac{\partial \Psi}{\partial \mathbf{u}}\right)^{T}
$$

Thus, Eq.(B.25) is equivalent to Eq.(B.4).

\section{References}

[1] V. G. Veselago, The electrodynamics of substances with simultaneously negative values of $\epsilon$ and $\mu$, Soviet Physics Uspekhi, 10 (4) (1968) 509-514.

[2] J. B. Pendry, A. J. Holden, W. J. Stewart and I. Youngs, Extremely low frequency plasmons in metallic mesostructures, Physical Review Letters, 76 (25) (1996) 4773-4776. 
[3] J. B. Pendry, A. J. Holden, D. J. Robbins and W. J. Stewart, Magnetism from conductors and enhanced nonlinear phenomena, IEEE Transactions on Microwave Theory and Techniques, 47 (11) (1999) 2075-2084.

[4] D. R. Smith, W. J. Padilla, D. C. Vier, S. C. Nemat-Nasser and S. Schultz, Composite medium with simultaneously negative permeability and permittivity, Physical Review Letters, 84 (18) (2000) 4184-4187.

[5] S. Hrabar, J. Bartolic and Z. Sipus, Waveguide minimization using uniaxial negative permeability metamaterial, IEEE Transactions on Antennas and Propagation, 53 (1) (2005) 110-119.

[6] D. Schurig, J. J. Mock, B. J. Justice, S. A. Cummer, J. B. Pendry, A. F. Starr, and D. R. Smith, Metamaterial electromagnetic cloak at microwave frequencies, Science, 314 (5801) (2006) 977-980.

[7] W. Cai, D. A. Genov and V. M. Shalaev, Superlens based on metal-dielectric composites, Physical Review B, 72 (19) (2005) 193101.

[8] C. Caloz, T. Itoh and A. Rennings, CRLH metamaterial leaky-wave and resonant antennas, IEEE Antennas and Propagation Magazine, 50 (5) (2008) $25-39$.

[9] Q. Cheng, T. J. Cui, W. X. Jiang and B. G. Cai, An omnidirectional electromagnetic absorber made of metamaterials, New Journal of Physics, 12 (2010) 063006.

[10] Q. Zhao, J. Zhou, F. Zhang and D. Lippens, Mie resonance-based dielectric metamaterials, Materials Today, 12 (12) (2009) 60-69.

[11] C. L. Holloway, E. F. Kuester, J. Baker-Jarvis and P. Kabos, A double negative (DNG) composite medium composed of magnetodielectric psherical particles embedded in a matrix, IEEE Transaction on Antennas and Propagation, 51 (10) (2003) 2596-2603.

[12] O. G. Vendik and M. S. Gashinova, Artificial double negative (DNG) media composed by two different dielectric sphere lattices embedded in a dielectric matrix, 34th European Microwave Conference, Amsterdam, Netherlands, 3 (2004) 1209-1212.

[13] A. Ahmadi and H. Mosallaei, Physical configuration and performance modeling of all-dielectric metamaterials, Physical Review B, 77 (4) (2008) 045104

[14] E. A. Semouchkina, G. B. Semouchkin, M. Lanagan and C. A. Randall, FDTD study of resonance processes in metamaterials, IEEE Transactions on Microwave Theory and Techniques, 53 (4) (2005) 1477-1487.

[15] Q. Zhao, L. Kang, B. Du, H. Zhao, Q. Xie, X. Huang, J. Zhou and L. Li, Experimental demonstration of isotropic negative permeability in a threedimensionial dielectric composite, Physical Review Letters, 101 (2) (2008) 027402 . 
[16] L. Peng, L. Ran, H. Chen, H. Zhang, J. A. Kong and T. M. Grzegorczyk, Experimental observation of left-handed behavior in an array of standard dielectric resonators, Physical Review Letters, 98 (15) (2007) 157403.

[17] K. Shibuya, K. Takano, N. Matsumoto, K.Izumi, H. Miyazaki, Y. Jimba and M. Hangyo, Terahertz metamaterials composed of $\mathrm{TiO}_{2}$ cube arrays, Proceedings of the 2nd International Congress on Advanced Electromagnetic Materials in Microwaves and Optics, Pamplona, Spain, September 21-26, 2008.,

[18] D. P. Gaillot, C. Croënne and D. Lippens, An all-dielectric route for terahertz cloaking, Optics Express, 16 (6) (2008) 3986-3992.

[19] W. Cai, U. K. Chettiar, A. V. Kildishev and V. M. Shalaev, Designs for optical cloaking with high-order transformations, Optics Express, 16 (8) (2008) 54445452 .

[20] T. Ueda, A. Lai and T. Itoh, Demonstration of negative refraction in a cutoff parallel-plate waveguide loaded with 2-D square lattice of dielectric resonators, IEEE Transactions on Microwave Theory and Techniques, 55 (6) (2007) 12801287.

[21] T. Ueda, N. Michishita, M. Akiyama and T. Itoh, Dielectric-resonator-based composite right/left-handed transmission lines and their application to leaky wave antenna, IEEE Transactions on Microwave Theory and Techniques, 56 (10) (2008) 2259-2269.

[22] A. J. Bensoussan, J. L. Lions and G. Papanicolau, Asymptotic Analysis for Periodic Structures, North-Holland Publishing Company, Amsterdam, 1978.

[23] E. Sanchez-Palencia, Non-homogeneous Media and Vibration Theory, Lecture Notes in Physics, Vol. 127, Springer-Verlag, Berlin, 1980.

[24] O. Ouchetto, C. W. Qiu, S. Zouhdi, L. W. Li and A. Razek, Homogenization of 3-D periodic bianisotropic metamaterials, IEEE Transactions on Microwave Theory and Techniques, 54 (11) (2006) 3893-3898.

[25] S. Zhou, W. Li and Q. Li, Design of 3-D periodic metamaterials for electromagnetic properties, IEEE Transactions on Microwave Theory and Techniques, 58 (4) (2010) 910-916.

[26] Z. Hashin, Analysis of composite materials - A survey, Journal of Applied Mechanics, 50 (3) (1983) 481-505.

[27] D. R. Smith and J. B. Pendry, Homogenization of metamaterials by field averaging, Journal of the Optical Society of America B, 23 (3) (2006) 391-403.

[28] D. R. Smith, S. Schultz, P. Markoš and C. M. Soukoulis, Determination of effective permittivity and permeability of metamaterials from reflection and transmission coefficients, Physical Review B, 65 (19) (2002) 195104.

[29] X. Chen, T. M. Grzegorczyk, B. I. Wu, J. Jr. Pacheco and J. A. Kong, Robust method to retrieve the constitutive effective parameters of metamaterials, Physical Review E, 70 (1) (2004) 016608. 
[30] G. Lubkowski, R. Schuhmann and T. Weiland, Extraction of effective metamaterial parameters by parameter fitting of dispersive models, Microwave and Optical Technology Letters, 49 (2) (2007) 285-288.

[31] D. R. Smith, D. C. Vier, T. Koschny and C. M. Soukoulis, Electromagnetic parameter retrieval from inhomogeneous metamaterials, Physical Review E, 71 (3) (2005) 036617.

[32] C. M. Soukoulis, S. Linden and M. Wegener, Negative refractive index at optical wavelengths, Science, 315 (5808) (2007), 47-49.

[33] S. Zhou, W. Li, G. Sun and Q. Li, A level-set procedure for the design of electromagnetic metamaterials, Optics Express, 18 (7) (2010) 6693-6702.

[34] S. Zhou, W. Li, Y. Chen, G. Sun and Q. Li, Topology optimization for negative permeability metamaterials using level-set algorithm, Acta Materialia, 59 (7) (2011) 2624-2636.

[35] S. Yamasaki, T. Nomura, K. Sato, N. Michishita, Y. Yamada and A. Kawamoto, Level set-based topology optimization targeting dielectric resonator-based composite right- and left-handed transmission lines, International Journal for Numerical Methods in Engineering, 89 (10) (2011) 1272-1295.

[36] M. Y. Wang, X. Wang and D. Guo, A level set method for structural topology optimization, Computer Methods in Applied Mechanics and Engineering, 192 (1-2) (2003) 227-246.

[37] G. Allaire, F. Jouve and A. M. Toader, Structural optimization using sensitivity analysis and a level-set method, Journal of Computational Physics, 194 (1) (2004) 363-393.

[38] M. P. Bendsøe and N. Kikuchi, Generating optimal topologies in structural design using a homogenization method, Computer Methods in Applied Mechanics and Engineering, 71 (2) (1988) 197-224.

[39] M. P. Bendsøe, Optimal shape design as a material distribution problem, Structural and Multidisciplinary Optimization, 1 (4) (1989) 193-202.

[40] K. Suzuki and N. Kikuchi, A homogenization method for shape and topology optimization, Computer Methods in Applied Mechanics and Engineering, 93 (3) (1991) 291-318.

[41] A. R. Diaz and N. Kikuchi, Solutions to shape and topology eigenvalue optimization problems using a homogenization method, International Journal for Numerical Methods in Engineering, 35 (7) (1992) 1487-1502.

[42] J. Yoo, N. Kikuchi and J. L. Volakis, Structural optimization in magnetic devices by the homogenization design method, IEEE Transactions on Magnetics, 36 (3) (2000) 574-580.

[43] A. R. Diaz and O. Sigmund, A topology optimization method for design of negative permeability metamaterials, Structural and Multidisciplinary Optimization, 41 (2) (2010) 163-177. 
[44] O. Sigmund, Systematic design of metamaterials by topology optimization, IUTAM Symposium on Modelling Nanomaterials and Nanosystems, R. Pyrz and J. C. Rauhe (eds.), Springer, Netherlands, 13 (2009) 151-159.

[45] J. S. Choi and J. Yoo, Design and application of layered composites with the prescribed magnetic permeability, International Journal for Numerical Methods in Engineering, 82 (1) (2010) 1-25.

[46] O. Sigmund, Materials with prescribed constitutive parameters: An inverse homogenization, International Journal of Solids and Structures, 31 (17) (1994) $2313-2329$.

[47] Y. El-Kahlout and G. Kiziltas, Inverse synthesis of electromagnetic materials using homogenization based topology optimization, Progress In Electromagnetics Research, 115 (2011) 343-380.

[48] M. Otomori, J. Andkjær, O. Sigmund, K. Izui, and S. Nishiwaki, Inverse design of dielectric materials by topology optimization, Progress In Electromagnetics Research, 127 (2012) 93-120.

[49] P. Y. Chen, C. H. Chen, H. Wang, J. H. Tsai and W. X. Ni, Synthesis design of artificial magnetic metamaterials using a genetic algorithm, Optics Express, 16 (17) (2008) 12806-12818.

[50] J. A. Bossard, S. Yun, D. H. Werner and T. S. Mayer, Synthesizing low loss negative index metamaterial stacks for the mid-infrared using genetic algorithms, Optics Express, 17 (17) (2009) 14771-14779.

[51] W. Wang, W. K. Xu and H. Hai, An effective method for designing new structural left-handed material based on topology optimisation, European Physical Journal-Applied Physics, 53 (2) (2011) 20401.

[52] F. Murat, L. Tartar, Optimality conditions and homogenization, Proceedings of Nonlinear Variational Problems, Pitman Publishing Program, Boston, (1985) $1-8$.

[53] J. K. Guest, J. H. Prévost and T. Belytschko, Achieving minimum length scale in topology optimization using nodal design variables and projection functions, International Journal for Numerical Methods in Engineering, 61 (2) (2004) 238254.

[54] O. Sigmund, Morphology-based black and white filters for topology optimization, Structural and Multidisciplinary Optimization, 33 (4-5) (2007) 401-424.

[55] J. K. Guest, Topology optimization with multiple phase projection, Computer Methods in Applied Mechanics and Engineering, 199 (1-4) (2009) 123-135.

[56] S. Xu, Y. Cai and G. Cheng, Volume preserving nonlinear density filter based on heaviside functions, Structural and Multidisciplinary Optimization, 41 (4) (2010) 495-505. 
[57] A. Kawamoto, T. Matsumori, S. Yamasaki, T. Nomura, T. Kondoh and S. Nishiwaki, Heaviside projection based topology optimization by a PDE-filtered scalar function, Structural and Multidisciplinary Optimization, 44 (1) (2011) $19-24$.

[58] F. Wang, B. S. Lazarov and O. Sigmund, On projection methods, convergence and robust formulations in topology optimization, Structural and Multidisciplinary Optimization, 43 (6) (2011) 767-784.

[59] P. Wei and M. Y. Wang, Piecewise constant level set method for structural topology optimization, International Journal for Numerical Methods in Engineering, 78 (4) (2009) 379-402.

[60] T. Yamada, K. Izui, S. Nishiwaki and A. Takezawa, A topology optimization method based on the level set method incorporating a fictitious interface energy, Computer Methods in Applied Mechanics and Engineering, 199 (45-48) (2010) $2876-2891$.

[61] T. Yamada, K. Izui and S. Nishiwaki, A level set-based topology optimization method for maximizing thermal diffusivity in problems including designdependent effects, Journal of Mechanical Design, 133 (3) (2011) 031011.

[62] J. M. Jin, and D. J. Riley, Finite Element Analysis of Antennas and Arrays, Wiley-IEEE Press, New Jersey, 2008.

[63] D. J. Bergman, The dielectric constant of a composite material - a problem in classical physics, Physics Reports, 43 (9) (1978) 377-407.

[64] R. T. Rockafellar, The multiplier method of Hestenes and Powell applied to convex programming, Journal of Optimization Theory and Applications, 12 (6) (1973) 555-562.

[65] J. Andkjær, S. Nishiwaki, T. Nomura and O. Sigmund, Topology optimization of grating couplers for the efficient excitation of surface plasmons, Journal of the Optical Society of America B-Optical Physics, 27 (9) (2010) 1828-1832.

[66] J. S. Jensen and O. Sigmund, Topology optimization of photonic crystal structures: a high-bandwidth low-loss T-junction waveguide, Journal of the Optical Society of America B-Optical Physics, 22 (6) (2005) 1191-1198. 


\section{List of Figures}

1 (a) Design domain and boundary conditions; (b) periodic structure for two-dimensional problem.

2 (a) Design domain and boundary conditions; (b) periodic structure for three-dimensional problem.

3 (a) A typical effective permeability curve; (b) effective permeability curve when real part of effective permeability is minimized directly; (c) $1^{\text {st }}$ stage; (d) $2^{\text {nd }}$ stage of two-stage optimization procedure.

4 Fixed design domain $D$ and level set function $\phi$.

5 Design domain and boundary conditions for two-dimensional problem.

6 Comparison of configurations from $1^{\text {st }}$ optimization stage for two-dimensional effective permeability minimization problem targeting 0.30THz: (a) initial; (b) optimized.

$7 \quad$ Effective permeability curves of $1^{\text {st }}$ optimization stage for two-dimensional effective permeability minimization problem targeting $0.30 \mathrm{THz}$.

8 Convergence history of objective function of $1^{\text {st }}$ optimization stage for two-dimensional effective permeability minimization problem targeting $0.30 \mathrm{THz}$.

9 Comparison of configurations from $2^{\text {nd }}$ optimization stage for two-dimensional effective permeability minimization problem targeting 0.30THz: (a) initial; (b) optimized.

10 Effective permeability curves for $2^{\text {nd }}$ optimization stage for two-dimensional effective permeability minimization problem targeting $0.30 \mathrm{THz}$.

11 Convergence history of objective function of $2^{\text {nd }}$ stage for two-dimensional effective permeability minimization problem targeting $0.30 \mathrm{THz}$.

12 Magnetic and electric field distributions for two-dimensional effective permeability minimization problem targeting 0.30THz: (a) initial; (b) optimized. 
13 Comparison of configurations for two-dimensional effective permeability minimization problem targeting $0.45 \mathrm{THz}$ : (a) initial; (b) optimized.

14 Effective permeability curves for two-dimensional effective permeability minimization problem targeting $0.45 \mathrm{THz}$.

15 Convergence histories of objective function for two-dimensional effective permeability minimization problem targeting $0.45 \mathrm{THz}$ : (a) $1^{\text {st }}$ optimization stage; (b) $2^{\text {nd }}$ optimization stage.

16 Magnetic and electric field distributions for two-dimensional effective permeability minimization problem targeting 0.45THz: (a) initial; (b) optimized.

17 Comparison of configurations for two-dimensional effective permeability design problem: (a) initial; (b) optimized.

18 Effective permeability curves for two-dimensional effective permeability design problem.

19 Convergence history of objective function of $2^{\text {nd }}$ optimization stage for two-dimensional effective permeability design problem.

20 Magnetic and electric field distributions for two-dimensional effective permeability minimization problem targeting 0.30THz: (a) initial; (b) optimized.

21 Comparison of configurations for two-dimensional effective permeability minimization problem targeting 0.30THz: (a) initial; (b) optimized.

22 Effective permeability curves for two-dimensional effective permeability minimization problem targeting $0.30 \mathrm{THz}$.

23 Convergence histories of objective function for two-dimensional effective permeability minimization problem targeting 0.30THz: (a) $1^{\text {st }}$ optimization stage; (b) $2^{\text {nd }}$ optimization stage.

24 Magnetic and electric field distributions for two-dimensional effective permeability minimization problem targeting 0.30THz: (a) initial; (b) optimized.

25 Comparison of configurations for two-dimensional effective permeability minimization problem targeting 0.45THz: (a) initial; (b) optimized. 
26 Effective permeability curves for two-dimensional effective permeability minimization problem targeting $0.45 \mathrm{THz}$.

27 Convergence histories of objective function for two-dimensional effective permeability minimization problem targeting 0.45THz: (a) $1^{\text {st }}$ optimization stage; (b) $2^{\text {nd }}$ optimization stage.

28 Magnetic and electric field distributions for two-dimensional effective permeability minimization problem targeting 0.45THz: (a) initial; (b) optimized.

29 Design domain and boundary conditions for three-dimensional design problem.

30 Comparison of configurations for three-dimensional effective permeability minimization problem targeting 0.30THz: (a) initial; (b) optimized.

31 Effective permeability curves for three-dimensional effective permeability minimization problem targeting $0.30 \mathrm{THz}$.

32 Convergence histories of objective function for threedimensional effective permeability minimization problem targeting 0.30THz: (a) $1^{\text {st }}$ optimization stage; (b) $2^{\text {nd }}$ optimization stage of the optimization.

33 Comparison of configurations for three-dimensional effective permeability minimization problem targeting $0.45 \mathrm{THz}$ : (a) initial; (b) optimized.

34 Effective permeability curves for three-dimensional effective permeability minimization problem targeting $0.45 \mathrm{THz}$.

35 Convergence histories of objective function for threedimensional effective permeability minimization problem targeting $0.45 \mathrm{THz}$ : (a) $1^{\text {st }}$ optimization stage; (b) $2^{\text {nd }}$ optimization stage. 


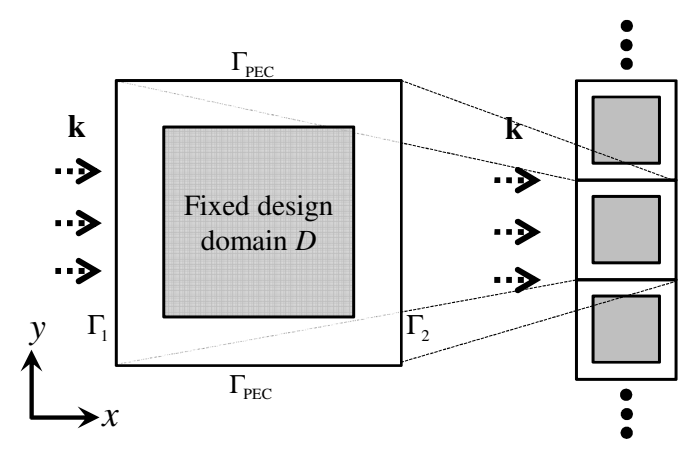

(a)

(b)

Fig. 1. (a) Design domain and boundary conditions; (b) periodic structure for two-dimensional problem. 


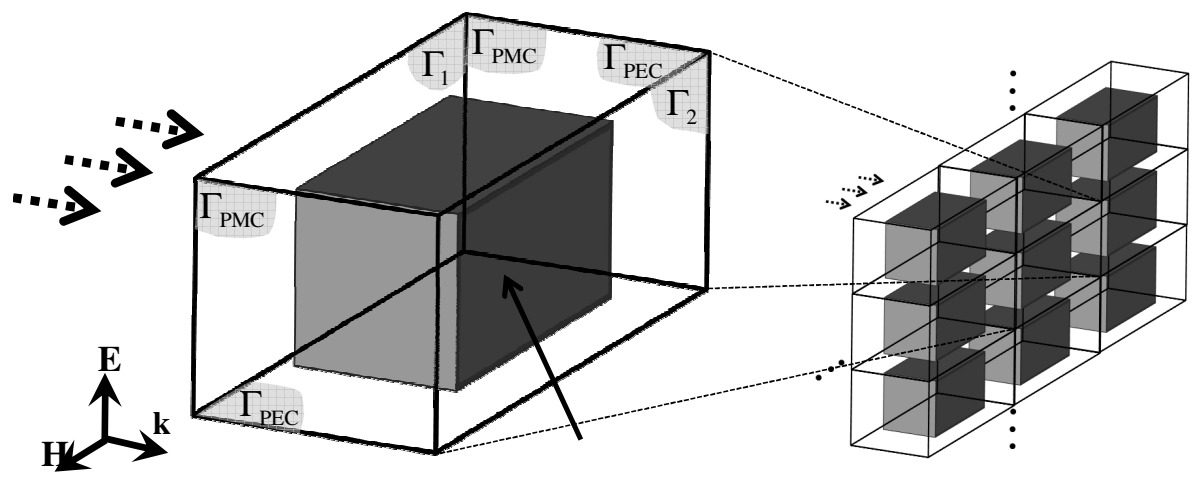

Fixed design domain $D$

(a)

(b)

Fig. 2. (a) Design domain and boundary conditions; (b) periodic structure for three-dimensional problem. 


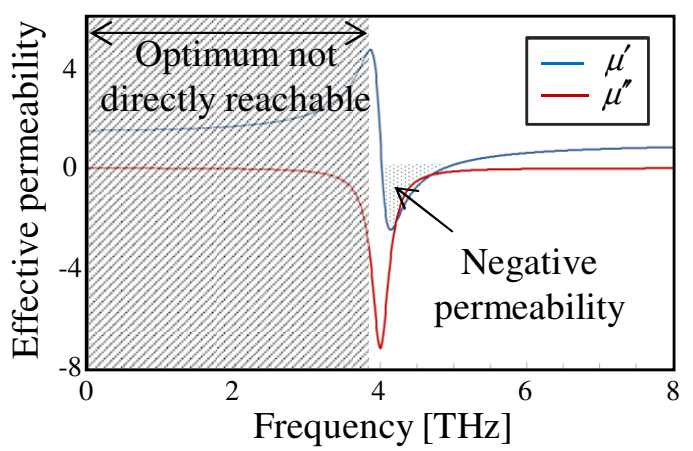

(a)

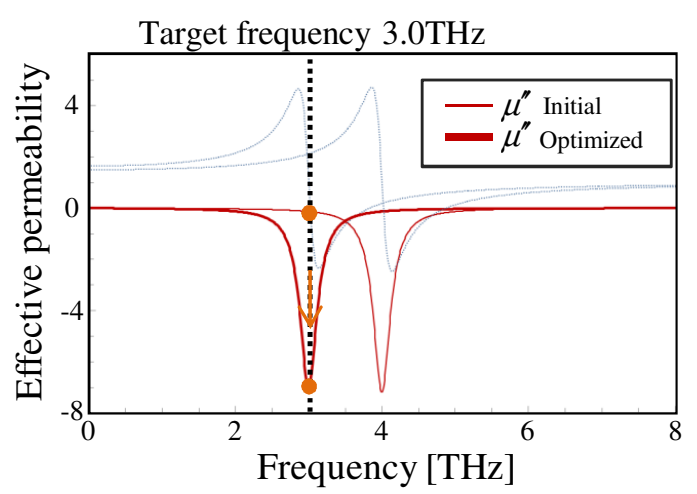

(c)

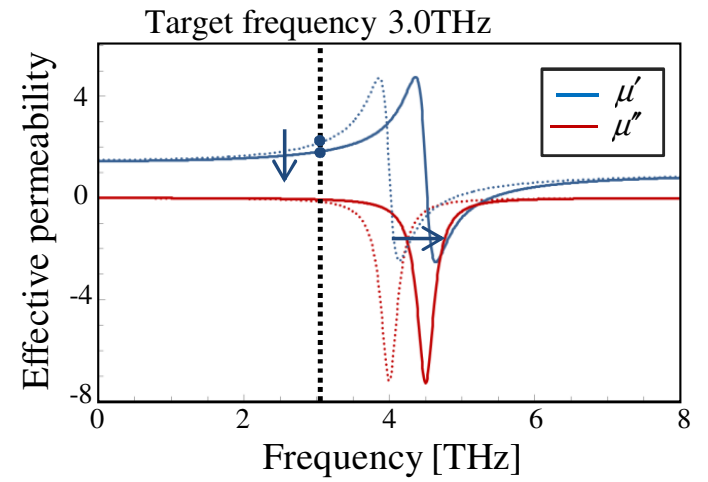

(b)

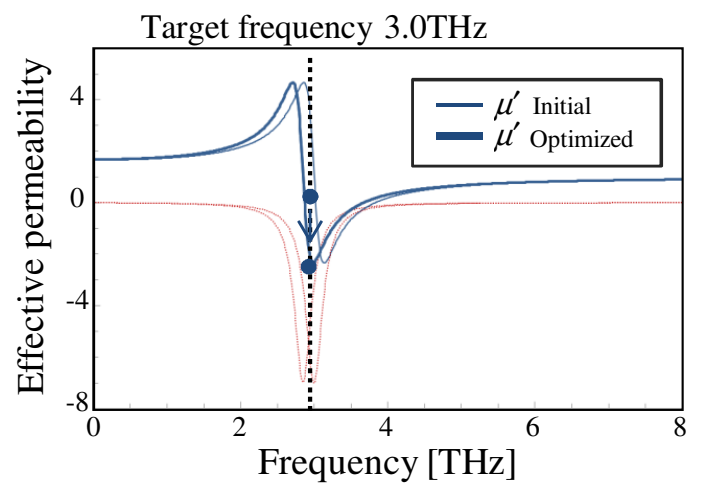

(d)

Fig. 3. (a) A typical effective permeability curve; (b) effective permeability curve when real part of effective permeability is minimized directly; (c) $1^{\text {st }}$ stage; (d) $2^{\text {nd }}$ stage of two-stage optimization procedure. 


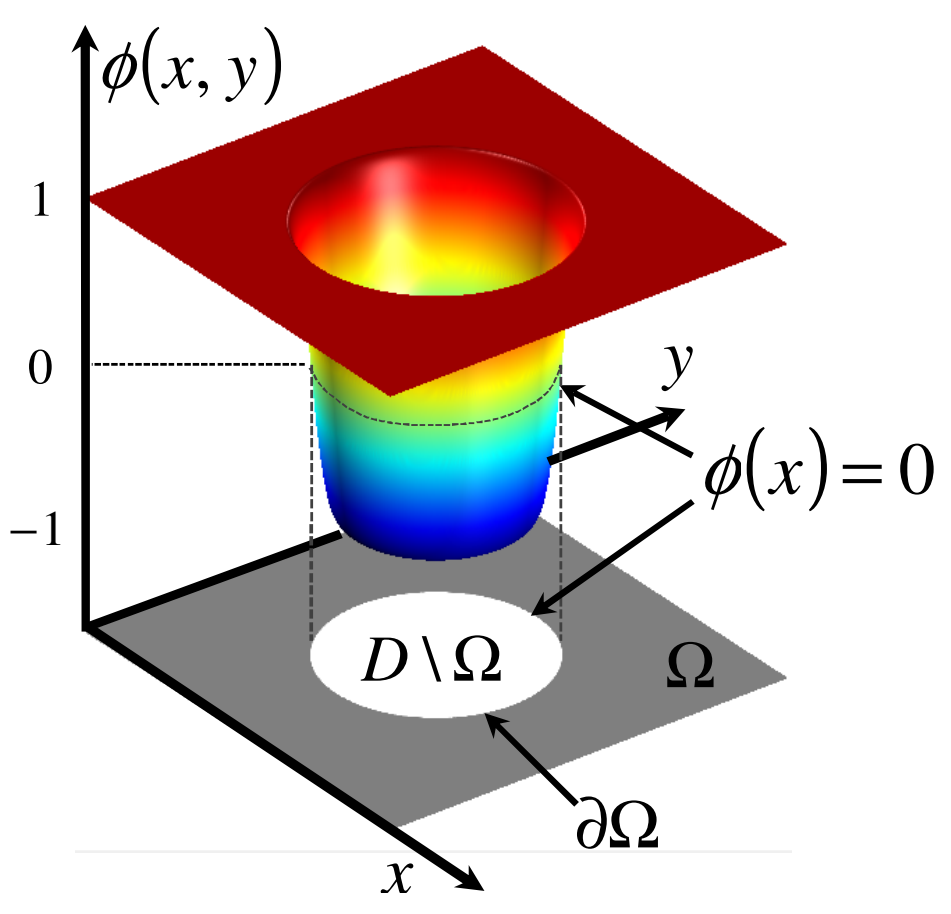

Fig. 4. Fixed design domain $D$ and level set function $\phi$. 


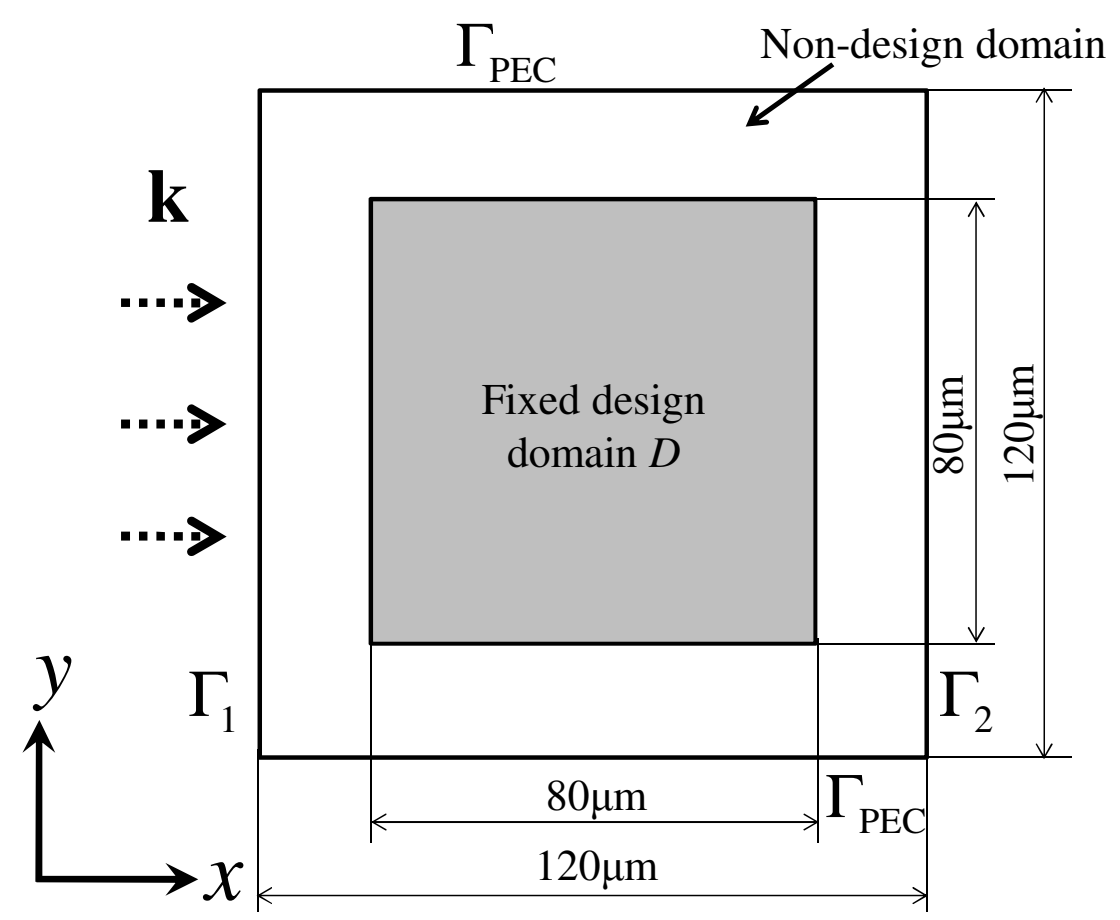

Fig. 5. Design domain and boundary conditions for two-dimensional problem. 


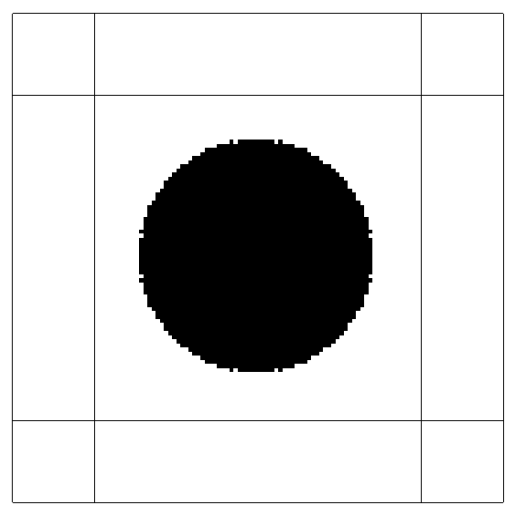

(a)

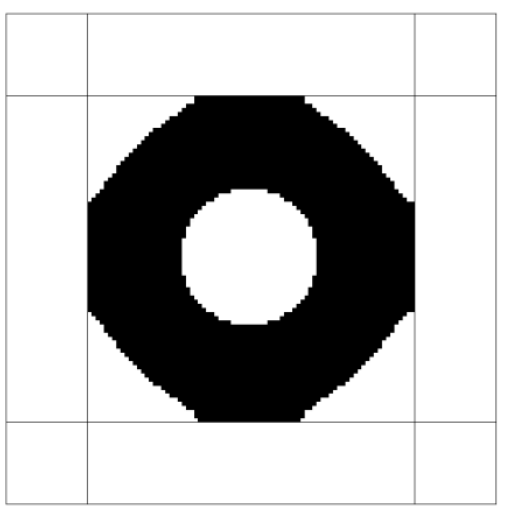

(b)

Fig. 6. Comparison of configurations from $1^{\text {st }}$ optimization stage for two-dimensional effective permeability minimization problem targeting 0.30THz: (a) initial; (b) optimized. 


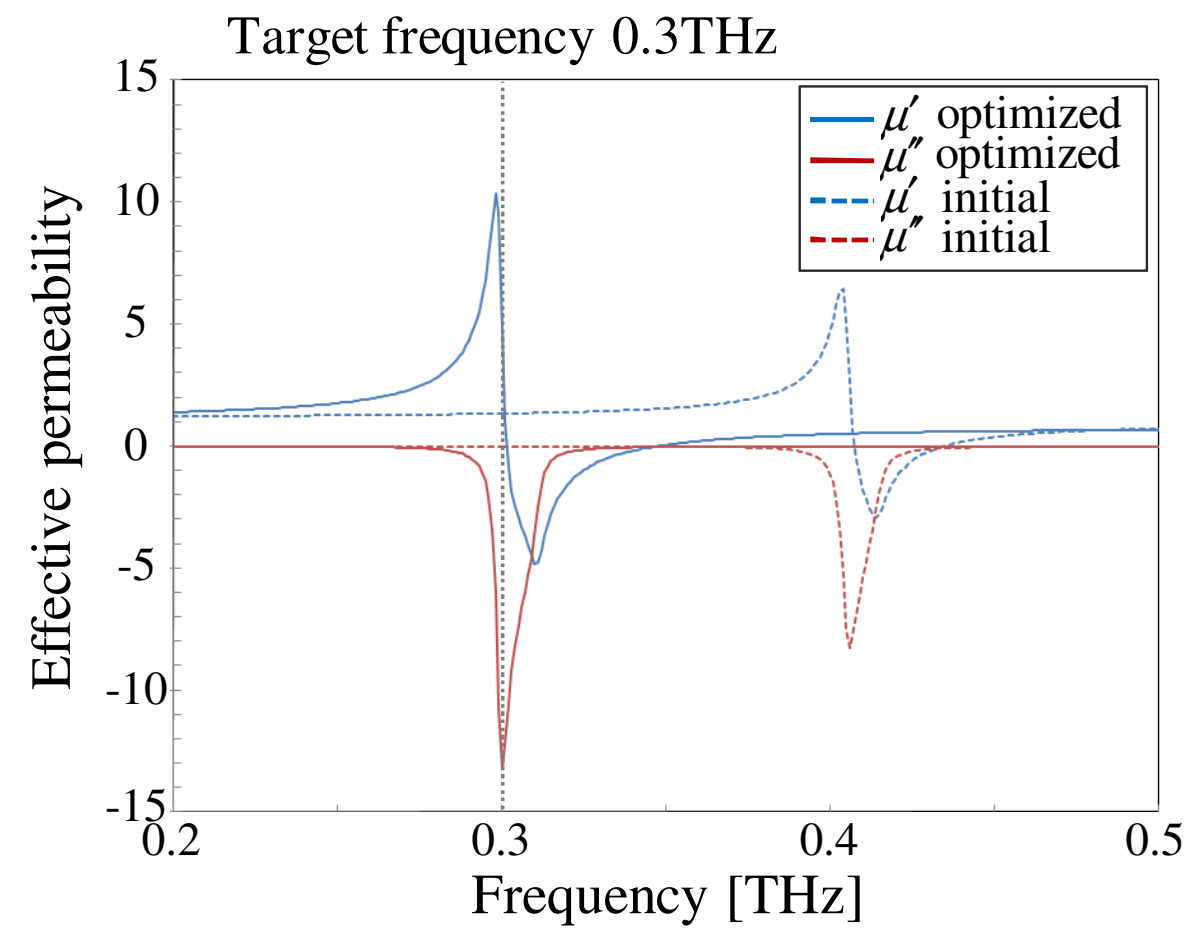

Fig. 7. Effective permeability curves of $1^{\text {st }}$ optimization stage for two-dimensional effective permeability minimization problem targeting $0.30 \mathrm{THz}$. 


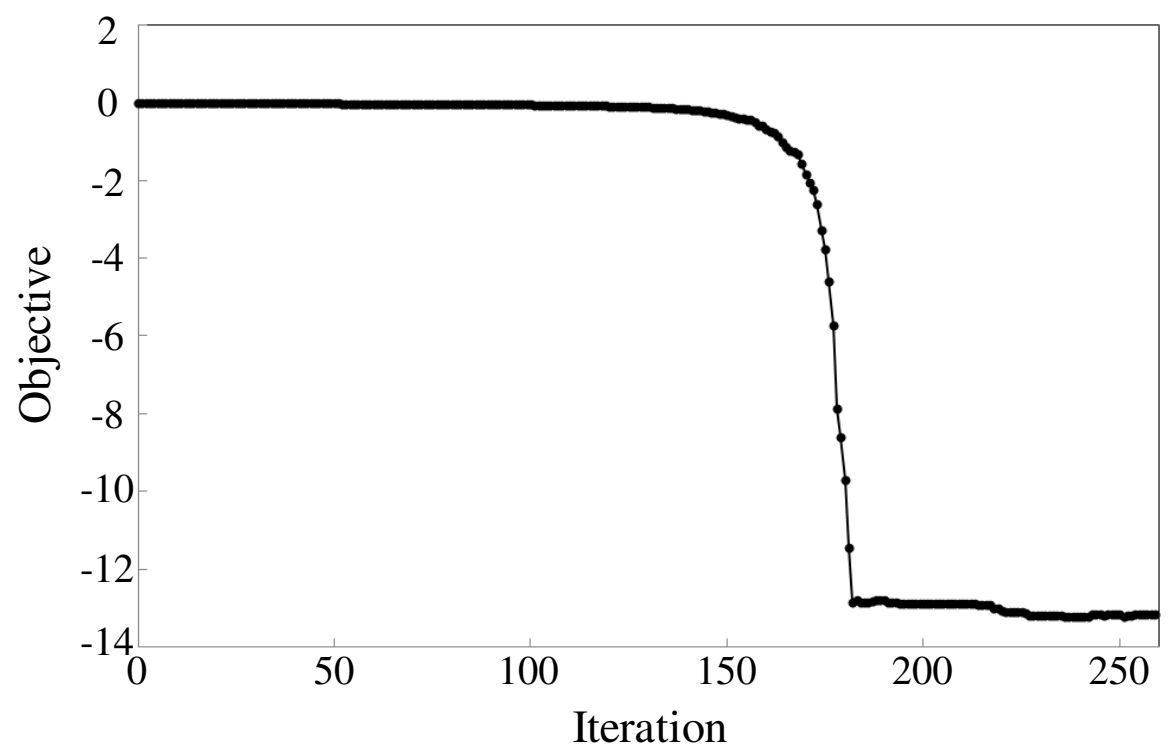

Fig. 8. Convergence history of objective function of $1^{\text {st }}$ optimization stage for two-dimensional effective permeability minimization problem targeting $0.30 \mathrm{THz}$. 


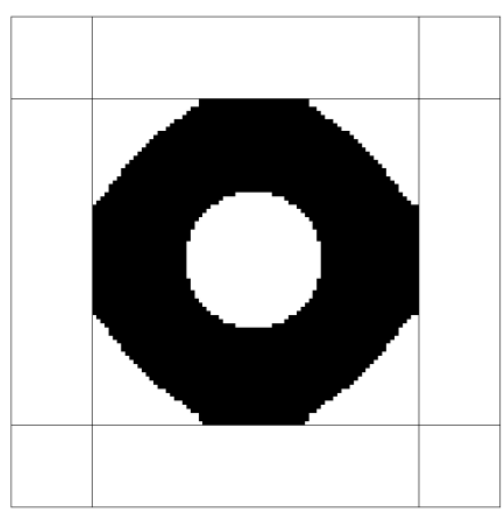

(a)

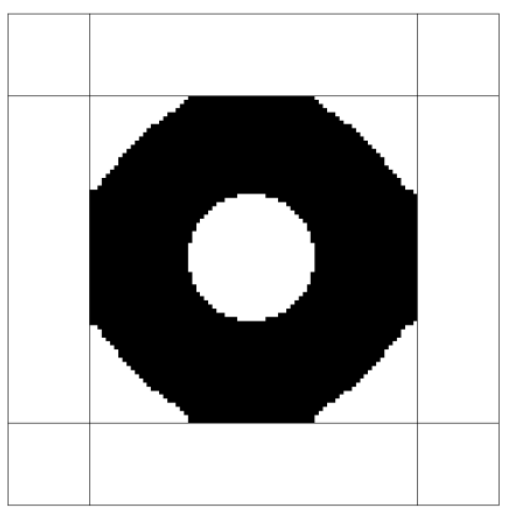

(b)

Fig. 9. Comparison of configurations from $2^{\text {nd }}$ optimization stage for two-dimensional effective permeability minimization problem targeting 0.30THz: (a) initial; (b) optimized. 


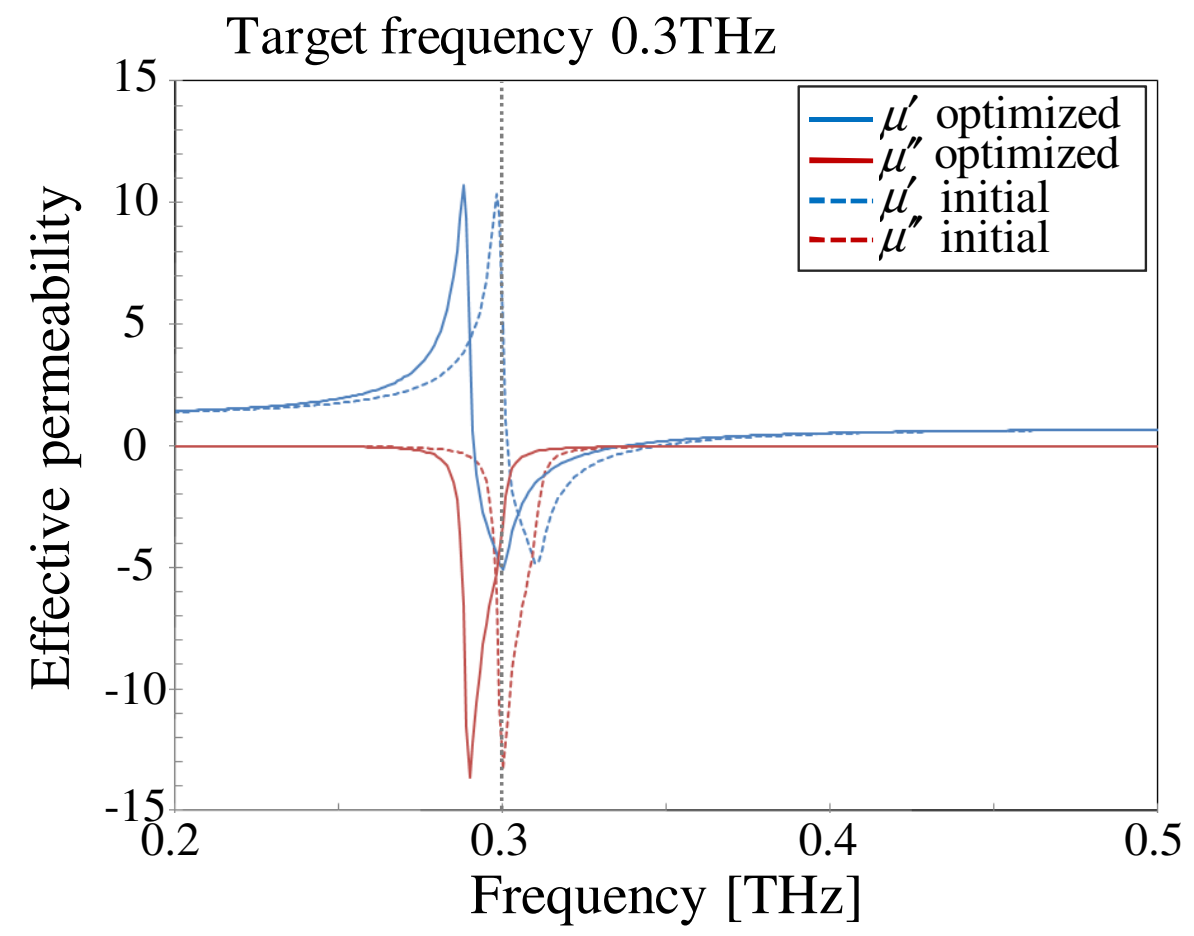

Fig. 10. Effective permeability curves for $2^{\text {nd }}$ optimization stage for two-dimensional effective permeability minimization problem targeting $0.30 \mathrm{THz}$. 


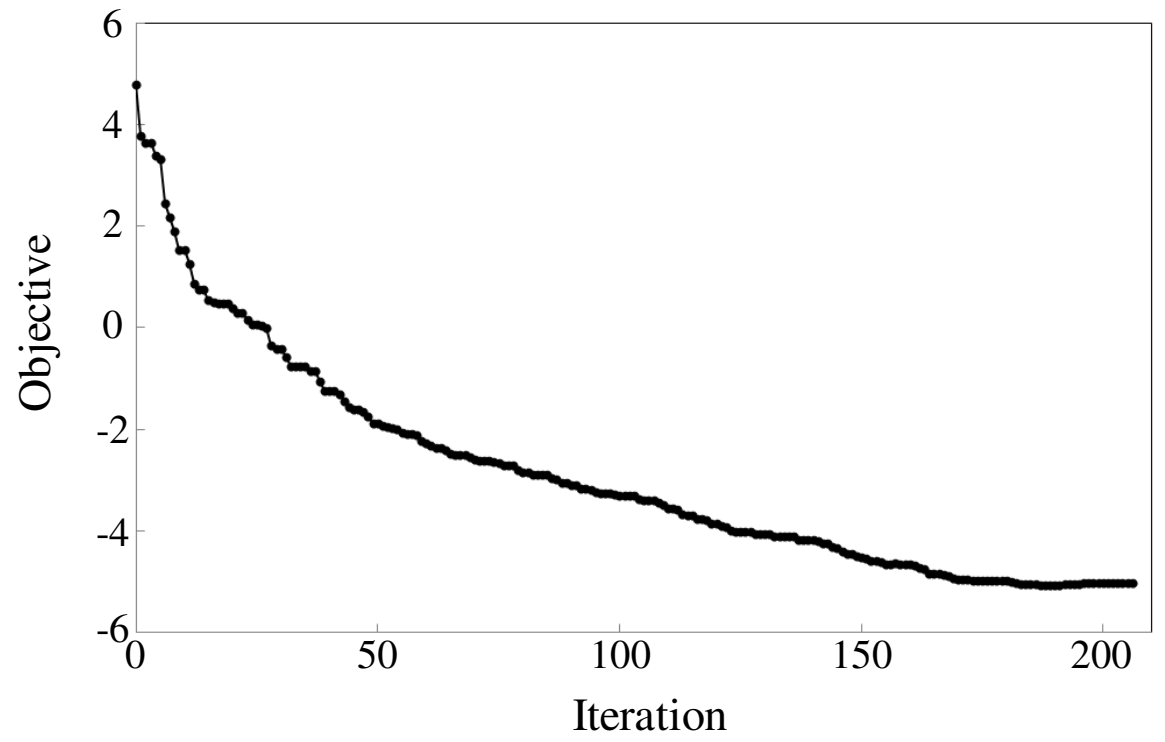

Fig. 11. Convergence history of objective function of $2^{\text {nd }}$ stage for two-dimensional effective permeability minimization problem targeting $0.30 \mathrm{THz}$. 


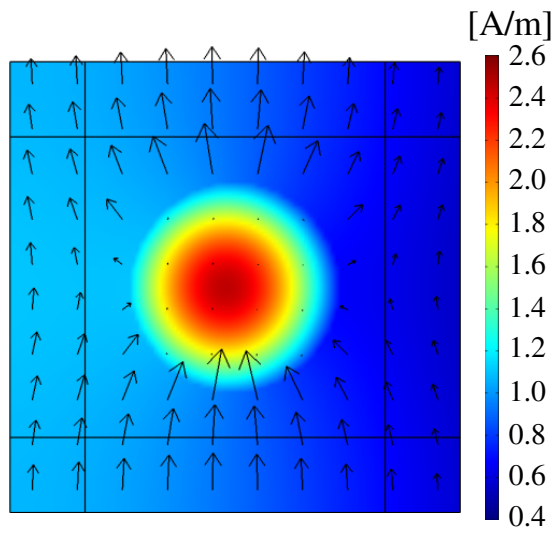

(a)

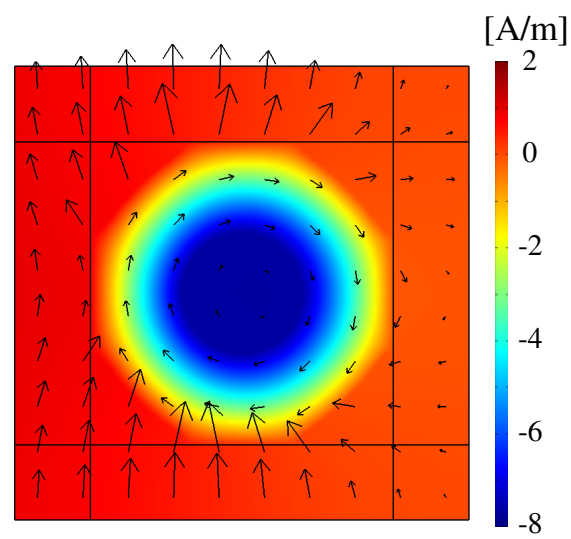

(b)

Fig. 12. Magnetic and electric field distributions for two-dimensional effective permeability minimization problem targeting $0.30 \mathrm{THz}$ : (a) initial; (b) optimized. 


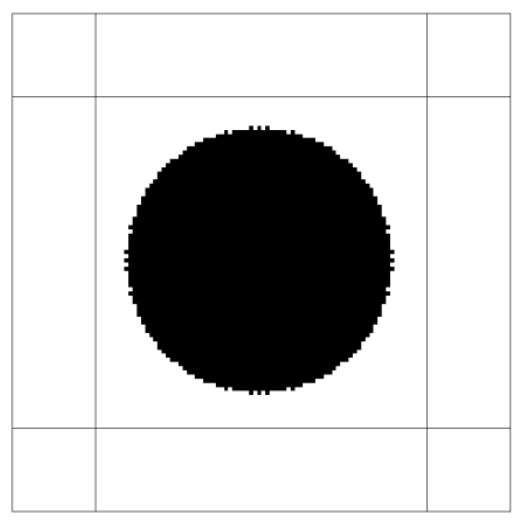

(a)

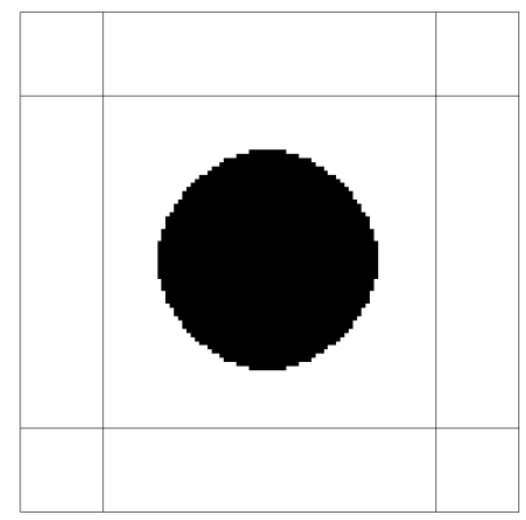

(b)

Fig. 13. Comparison of configurations for two-dimensional effective permeability minimization problem targeting $0.45 \mathrm{THz}$ : (a) initial; (b) optimized. 


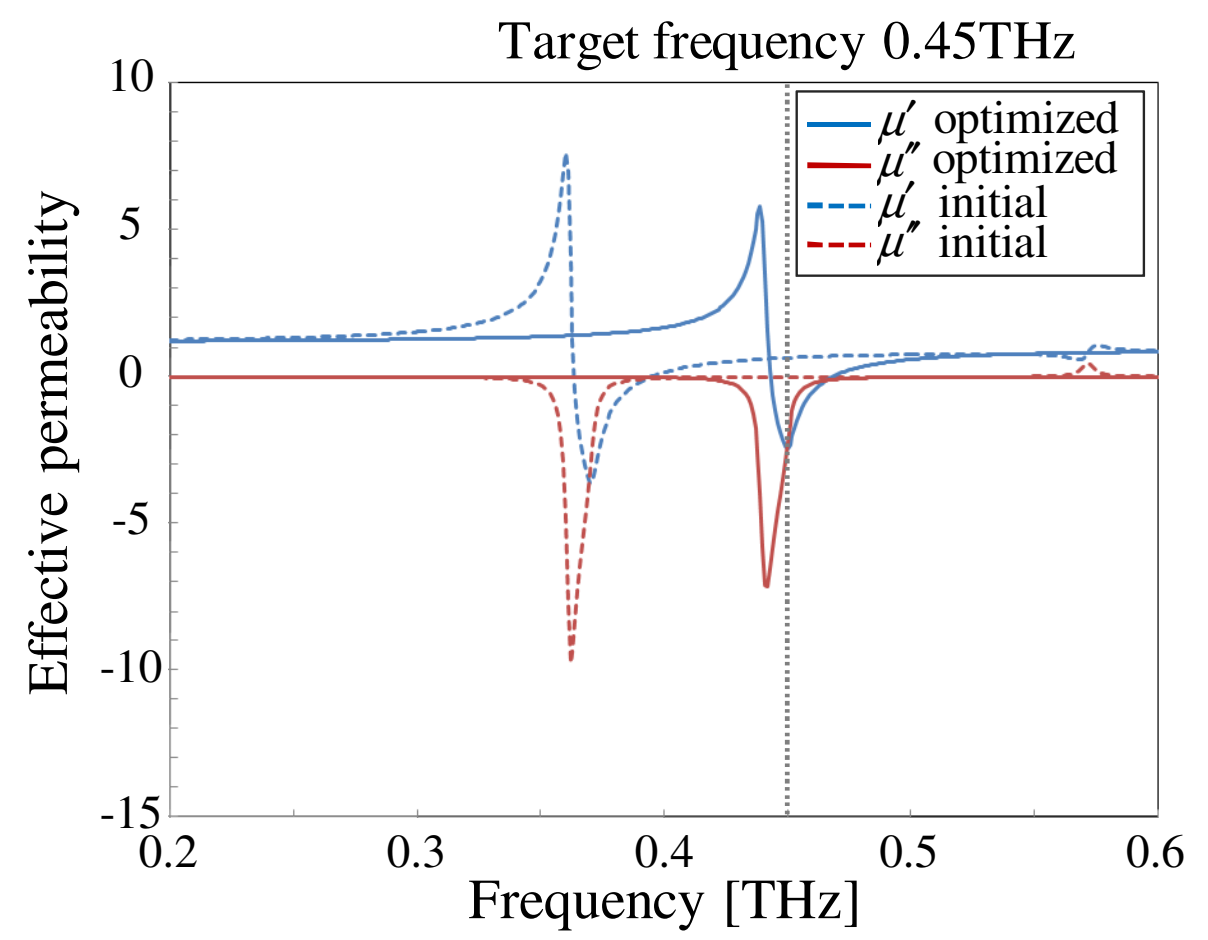

Fig. 14. Effective permeability curves for two-dimensional effective permeability minimization problem targeting $0.45 \mathrm{THz}$. 


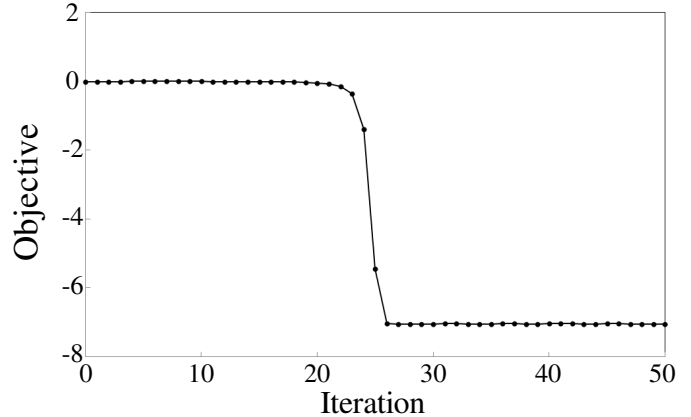

(a)

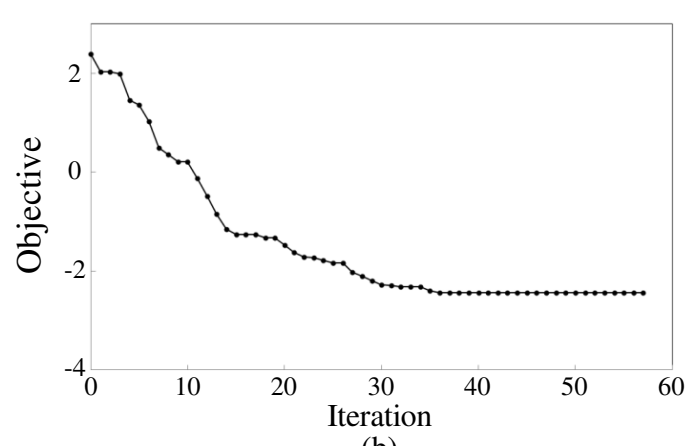

(b)

Fig. 15. Convergence histories of objective function for two-dimensional effective permeability minimization problem targeting $0.45 \mathrm{THz}$ (a) $1^{\text {st }}$ optimization stage; (b) $2^{\text {nd }}$ optimization stage. 


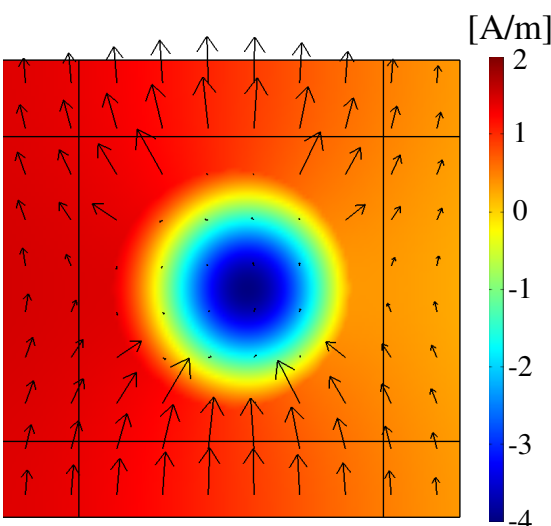

(a)

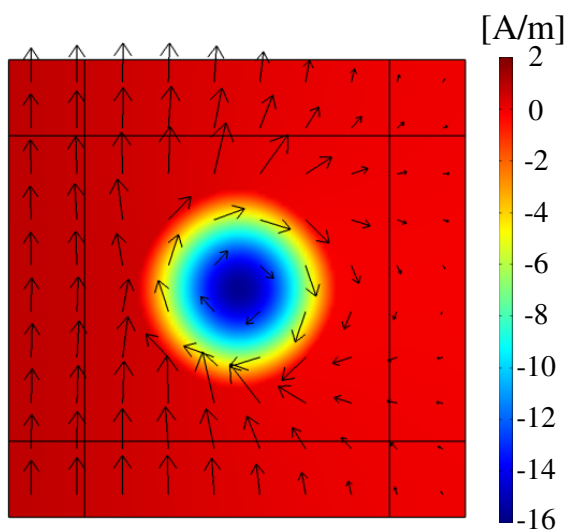

(b)

Fig. 16. Magnetic and electric field distributions for two-dimensional effective permeability minimization problem targeting $0.45 \mathrm{THz}$ : (a) initial; (b) optimized. 


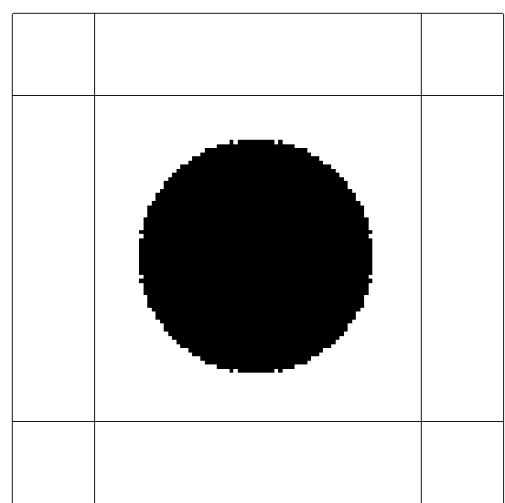

(a)

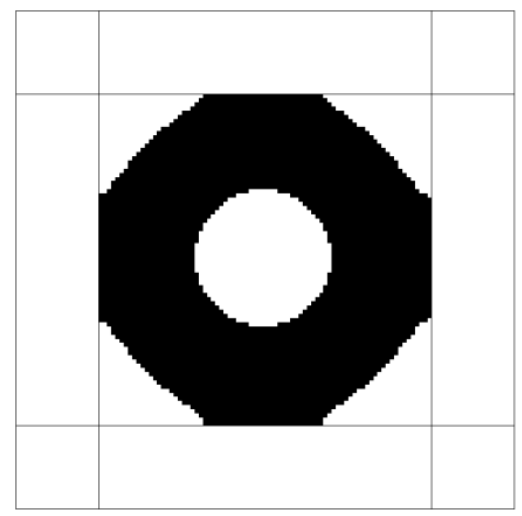

(b)

Fig. 17. Comparison of configurations for two-dimensional effective permeability design problem: (a) initial; (b) optimized. 


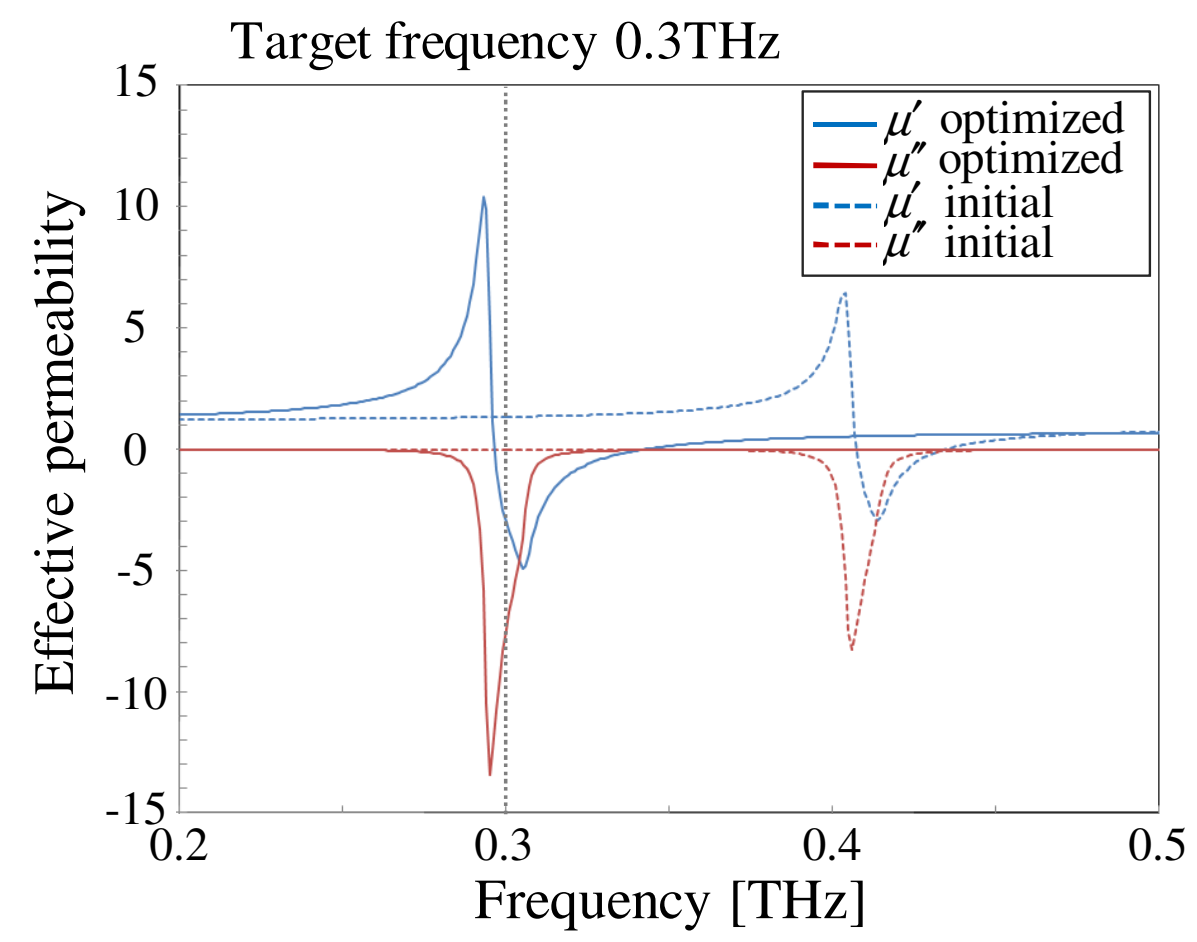

Fig. 18. Effective permeability curves for two-dimensional effective permeability design problem. 


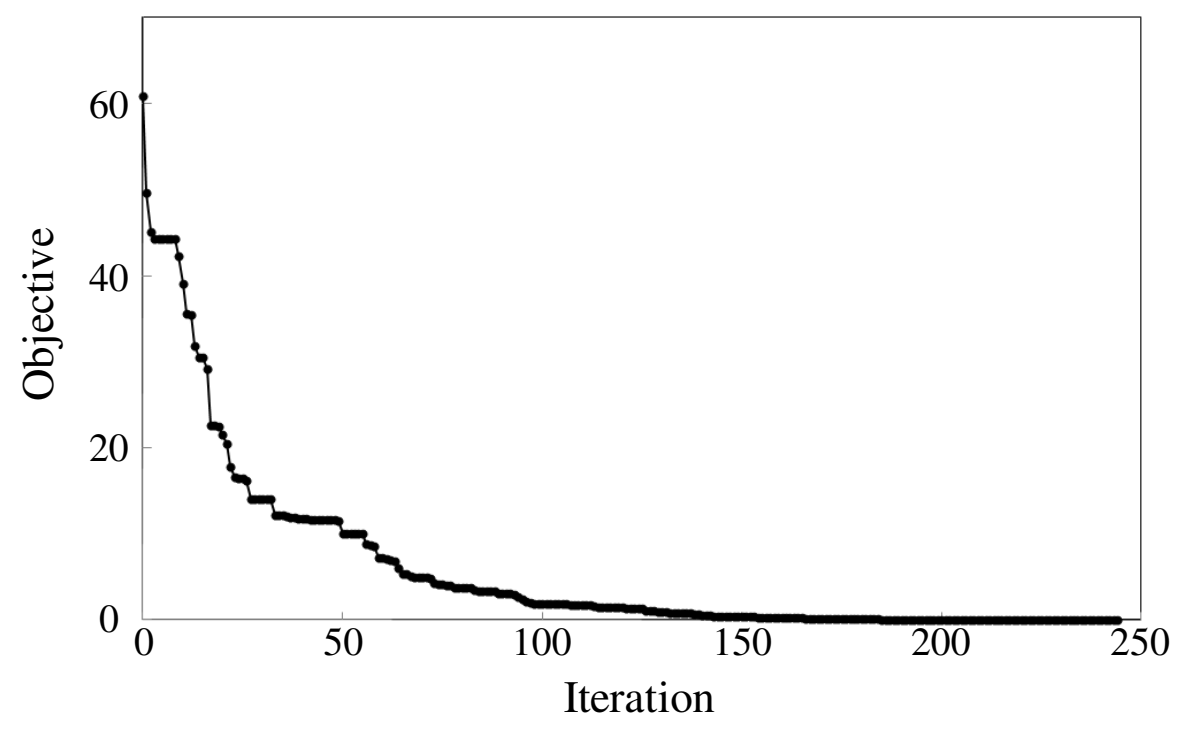

Fig. 19. Convergence history of objective function of $2^{\text {nd }}$ optimization stage for two-dimensional effective permeability design problem. 


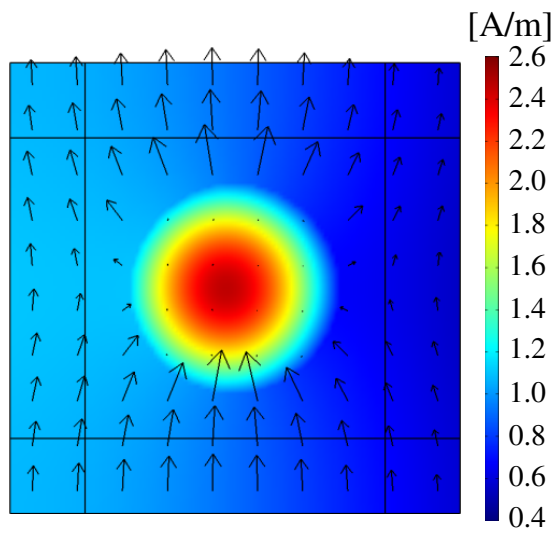

(a)

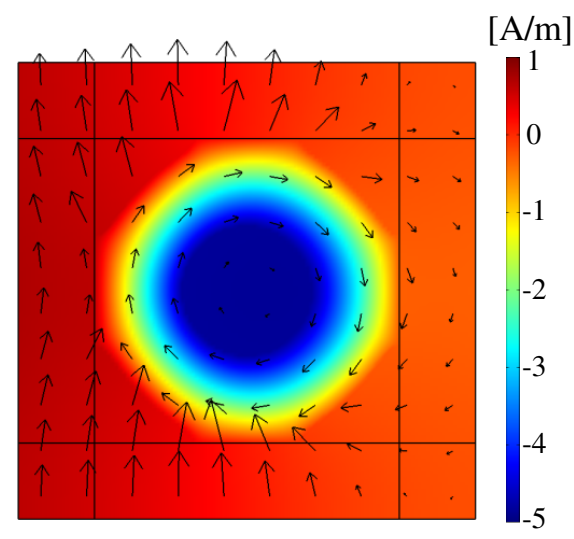

(b)

Fig. 20. Magnetic and electric field distributions for two-dimensional effective permeability minimization problem targeting $0.30 \mathrm{THz}$ : (a) initial; (b) optimized. 


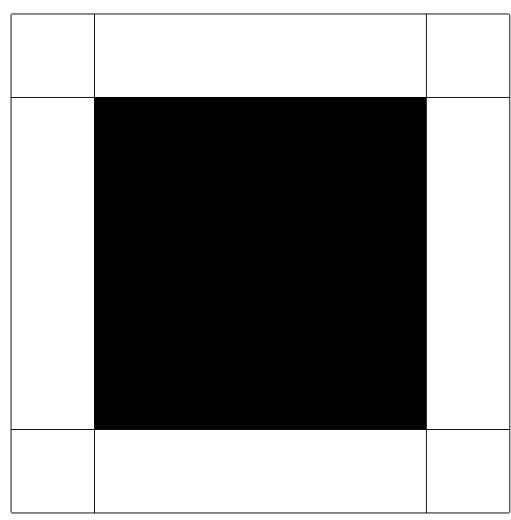

(a)

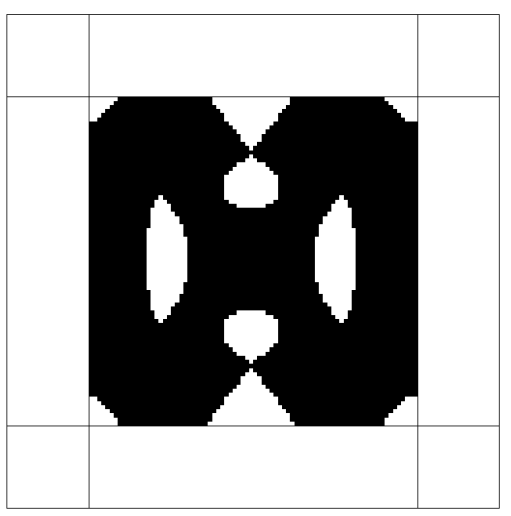

(b)

Fig. 21. Comparison of configurations for two-dimensional effective permeability minimization problem targeting 0.30THz: (a) initial; (b) optimized. 


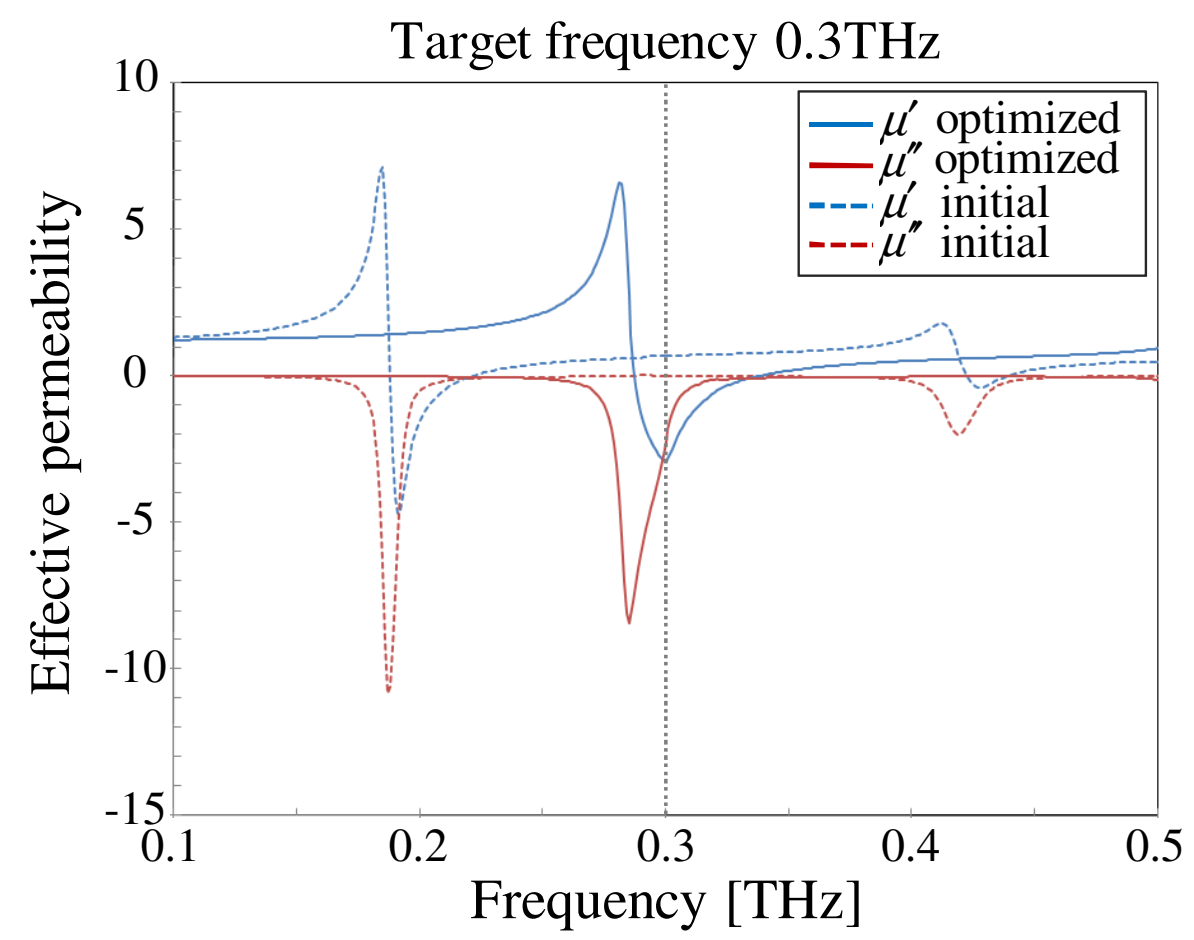

Fig. 22. Effective permeability curves for two-dimensional effective permeability minimization problem targeting $0.30 \mathrm{THz}$. 


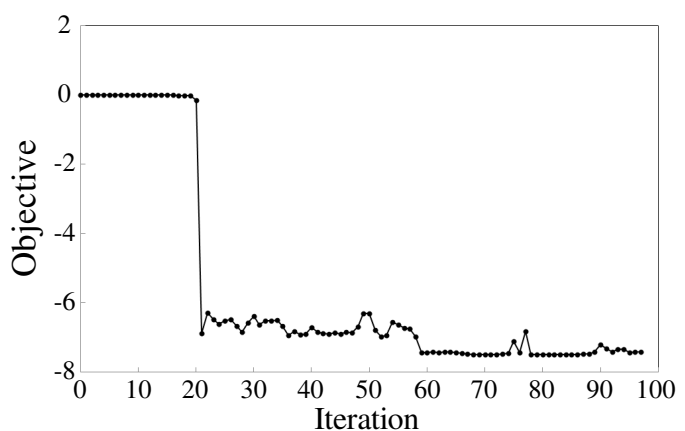

(a)

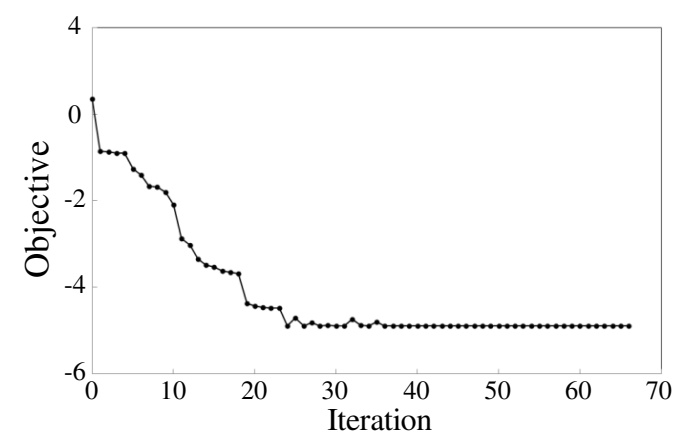

(b)

Fig. 23. Convergence histories of objective function for two-dimensional effective permeability minimization problem targeting $0.30 \mathrm{THz}$ (a) $1^{\text {st }}$ optimization stage; (b) $2^{\text {nd }}$ optimization stage. 


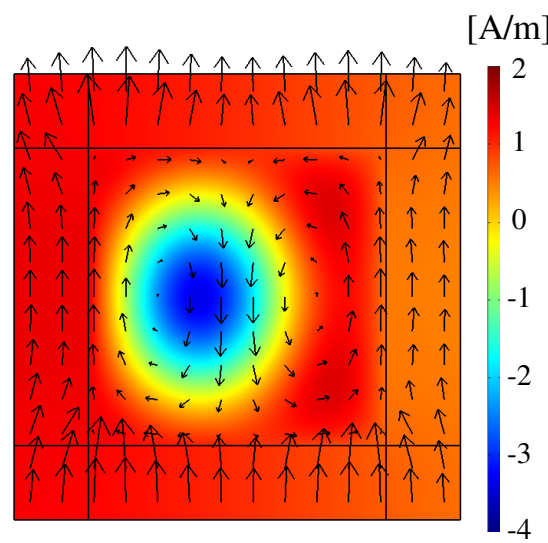

(a)

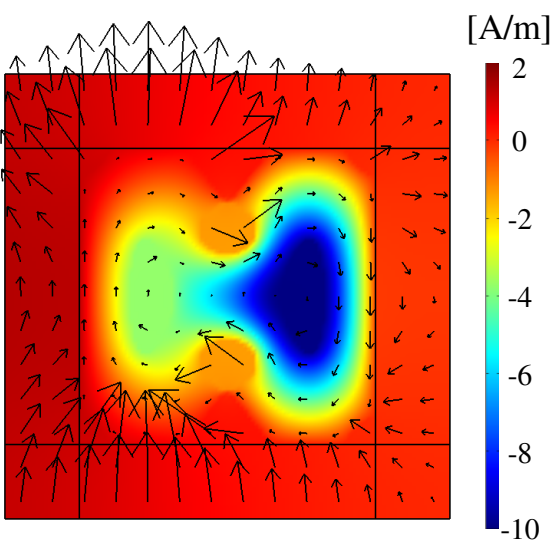

(b)

Fig. 24. Magnetic and electric field distributions for two-dimensional effective permeability minimization problem targeting $0.30 \mathrm{THz}$ : (a) initial; (b) optimized. 


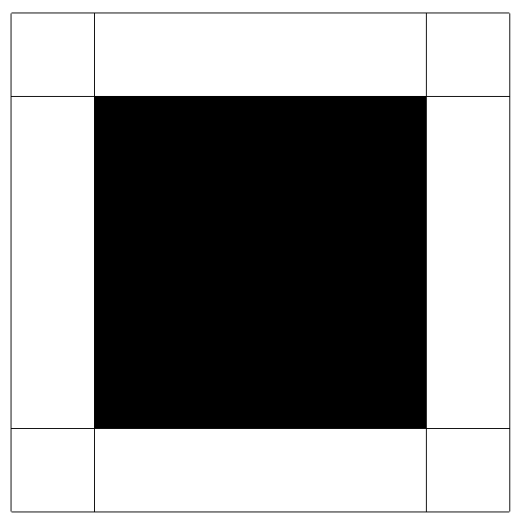

(a)

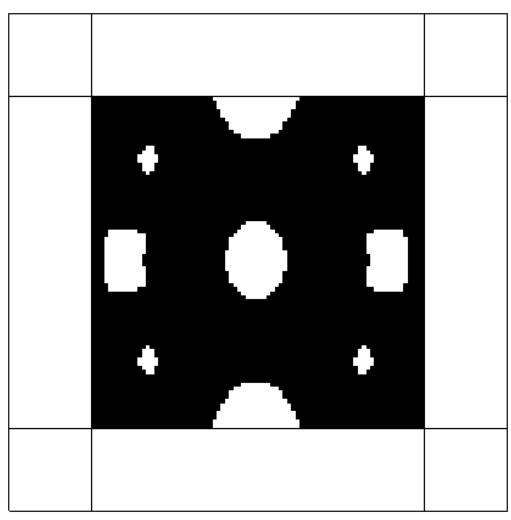

(b)

Fig. 25. Comparison of configurations for two-dimensional effective permeability minimization problem targeting $0.45 \mathrm{THz}$ : (a) initial; (b) optimized. 


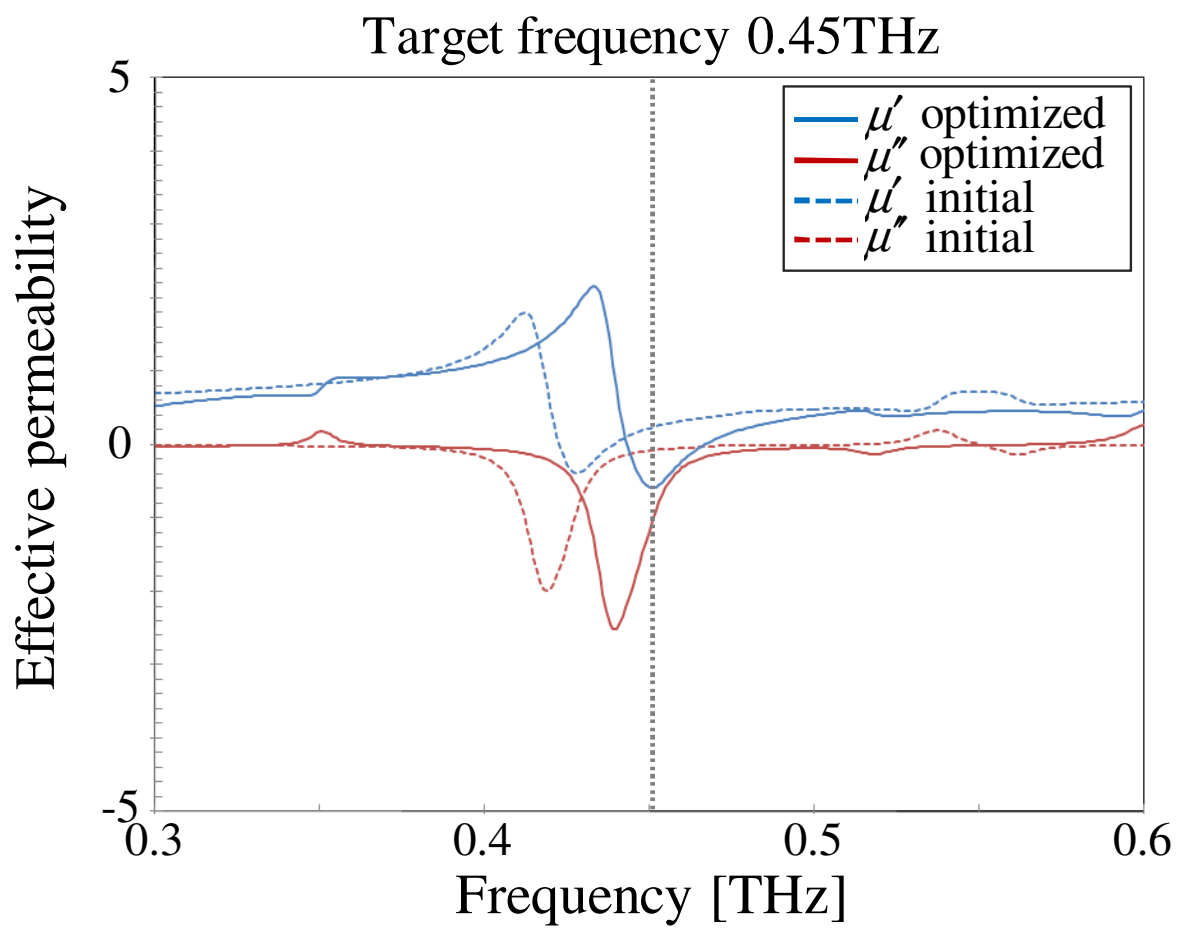

Fig. 26. Effective permeability curves for two-dimensional effective permeability minimization problem targeting $0.45 \mathrm{THz}$. 


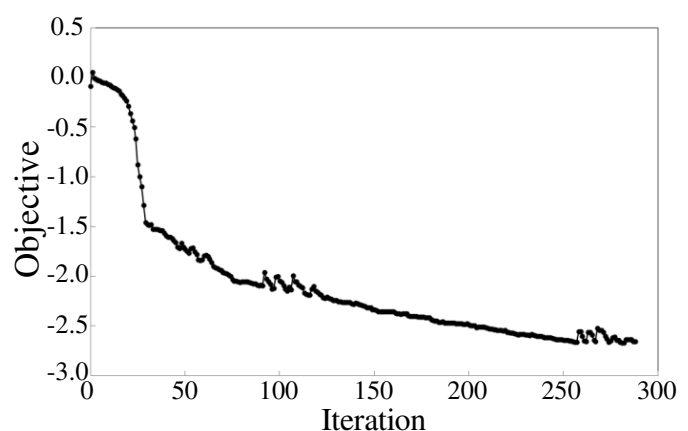

(a)

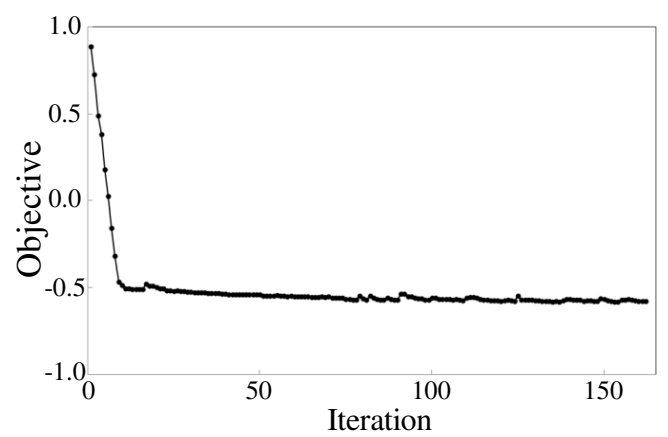

(b)

Fig. 27. Convergence histories of objective function for two-dimensional effective permeability minimization problem targeting $0.45 \mathrm{THz}$ : (a) $1^{\text {st }}$ optimization stage; (b) $2^{\text {nd }}$ optimization stage. 


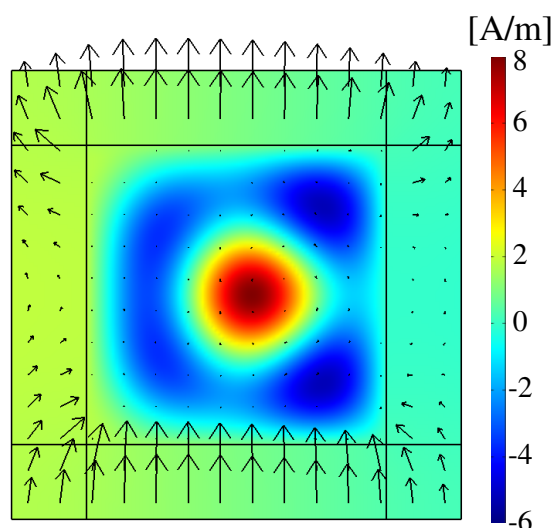

(a)

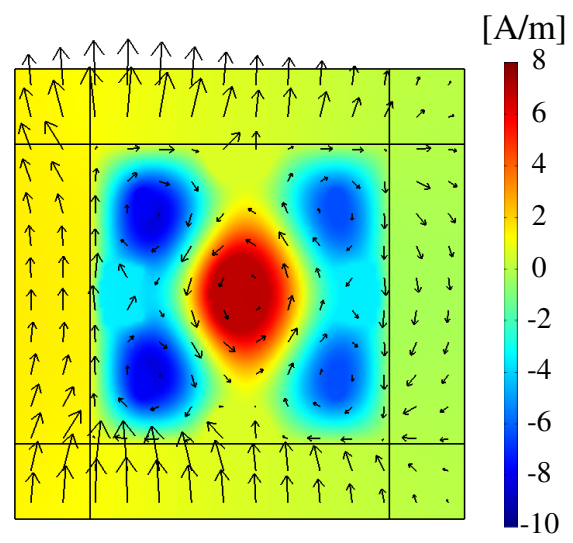

(b)

Fig. 28. Magnetic and electric field distributions for two-dimensional effective permeability minimization problem targeting $0.45 \mathrm{THz}$ : (a) initial; (b) optimized. 


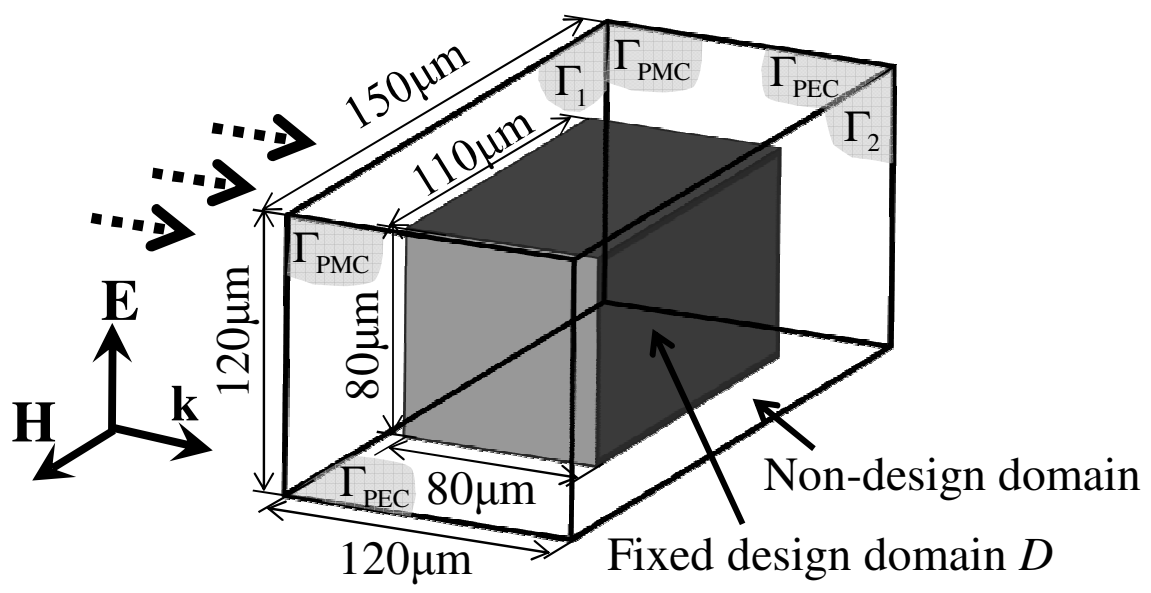

Fig. 29. Design domain and boundary conditions for three-dimensional design problem. 


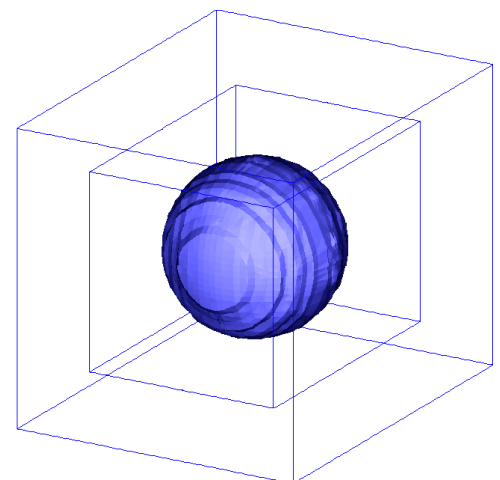

(a)

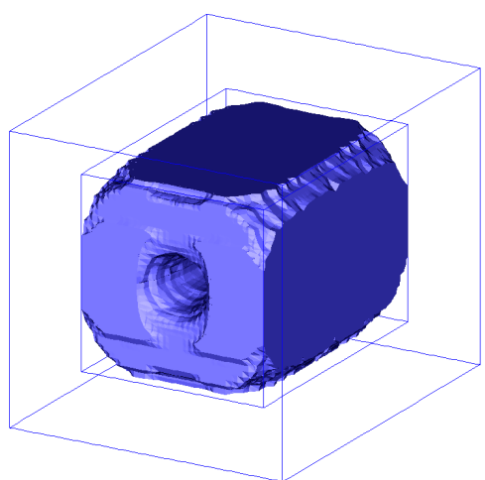

(b)

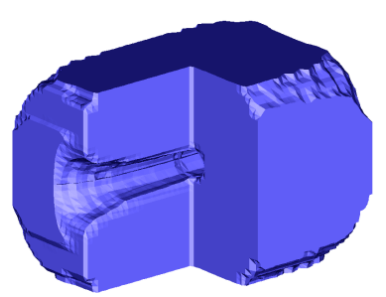

Fig. 30. Comparison of configurations for three-dimensional effective permeability minimization problem targeting 0.30THz: (a) initial; (b) optimized. 


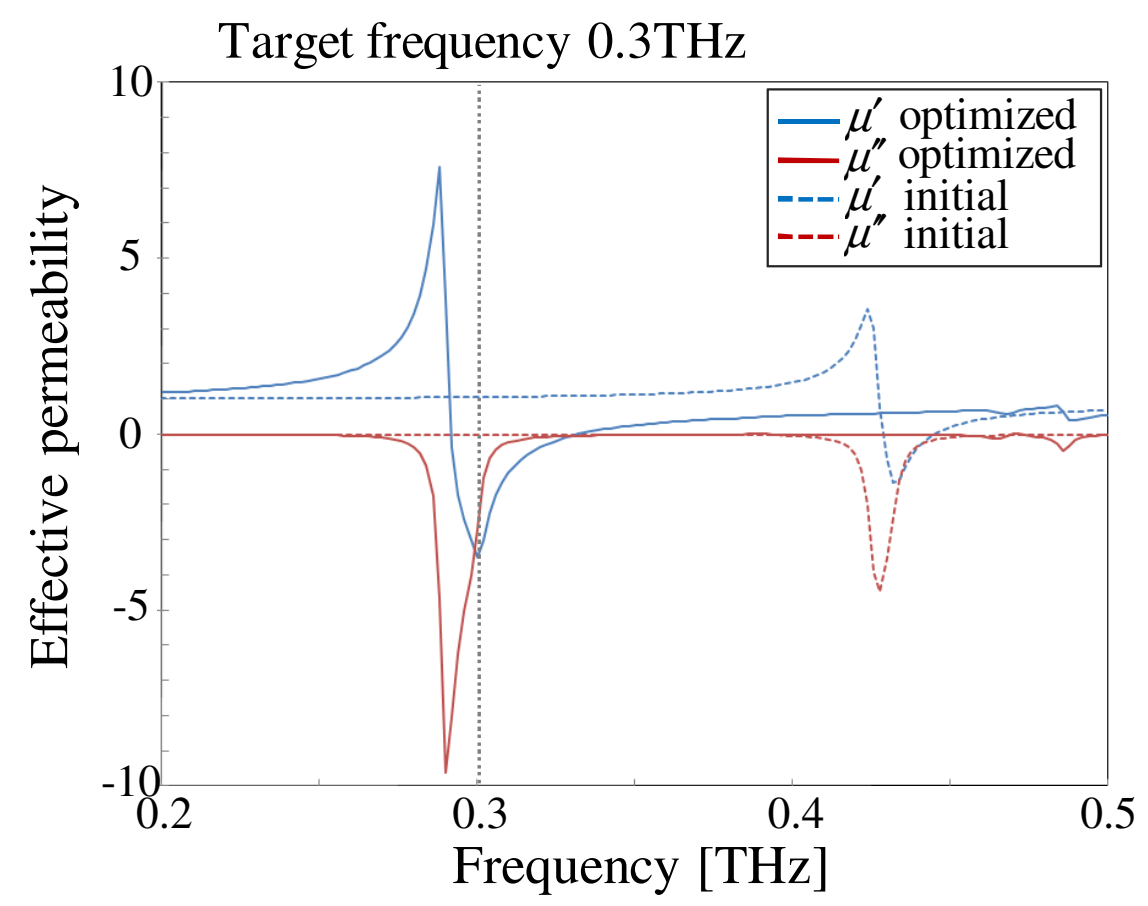

Fig. 31. Effective permeability curves for three-dimensional effective permeability minimization problem targeting $0.30 \mathrm{THz}$. 


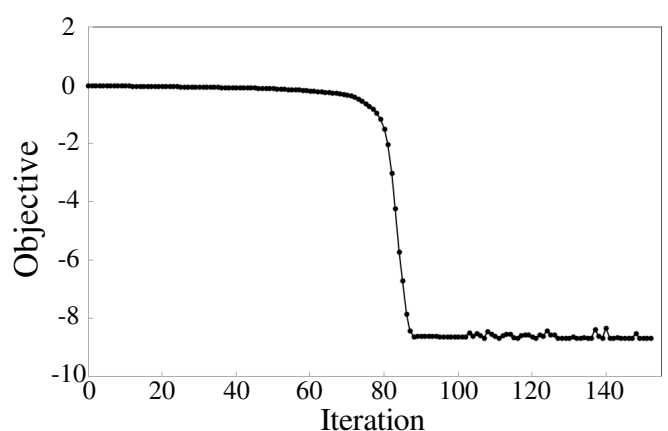

(a)

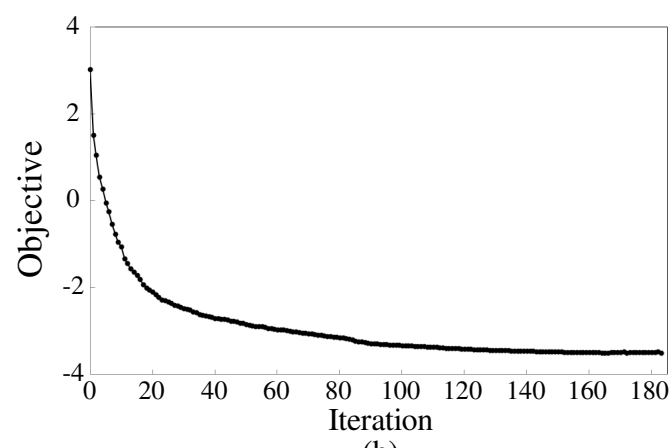

(b)

Fig. 32. Convergence histories of objective function for three-dimensional effective permeability minimization problem targeting $0.30 \mathrm{THz}$ : (a) $1^{\text {st }}$ optimization stage; (b) $2^{\text {nd }}$ optimization stage of the optimization. 


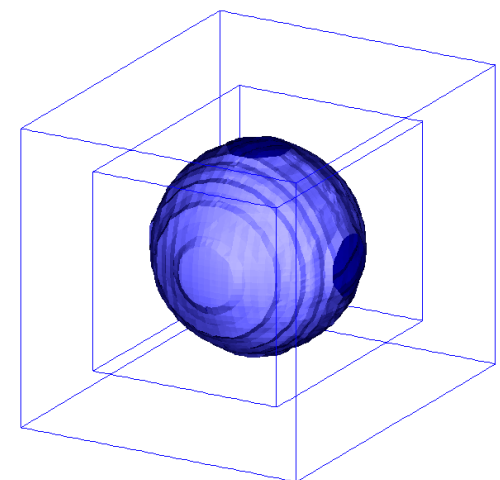

(a)

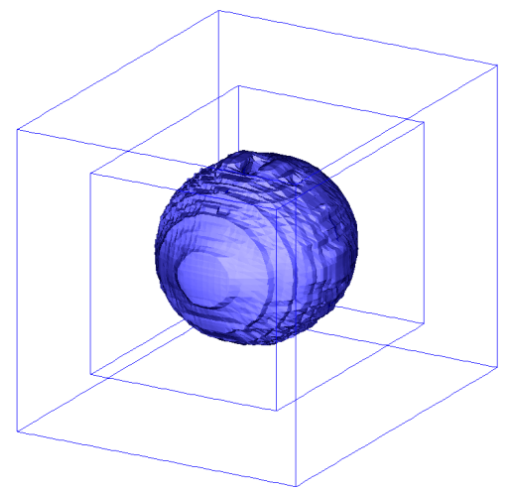

(b)

Fig. 33. Comparison of configurations for three-dimensional effective permeability minimization problem targeting $0.45 \mathrm{THz}$ : (a) initial; (b) optimized. 


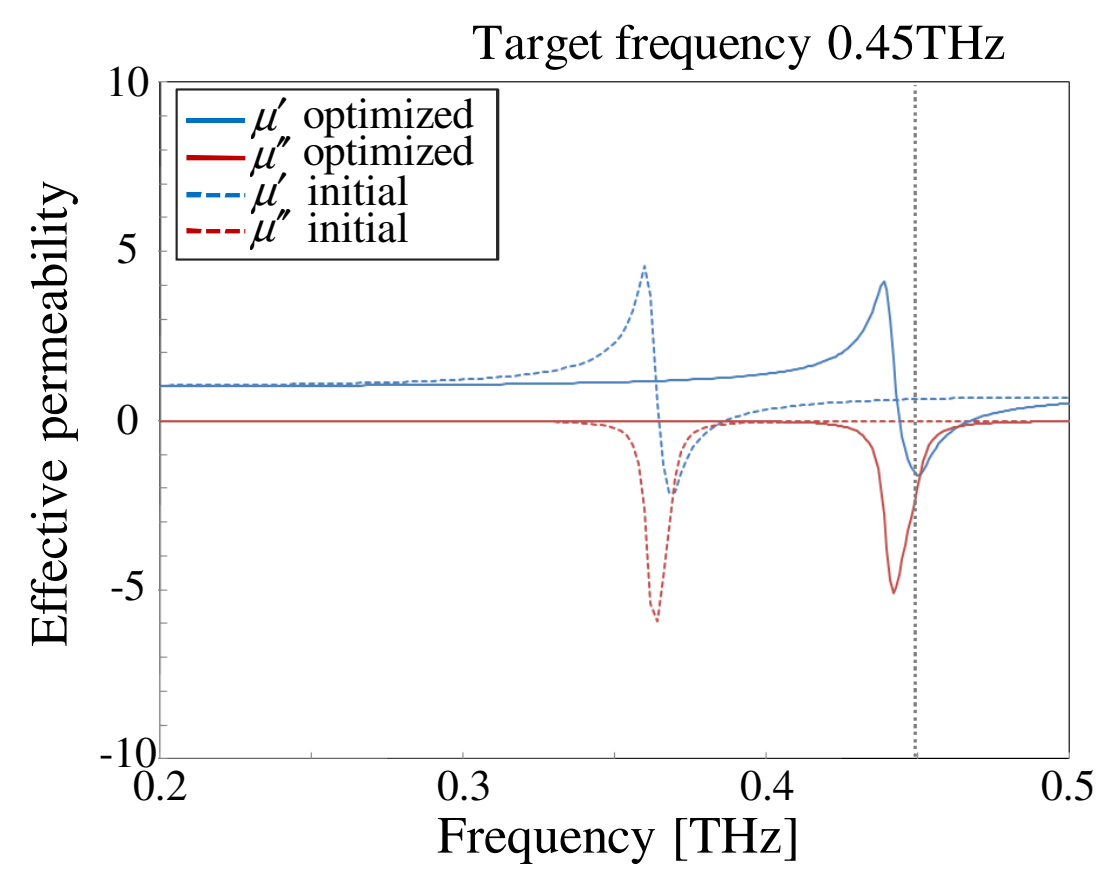

Fig. 34. Effective permeability curves for three-dimensional effective permeability minimization problem targeting $0.45 \mathrm{THz}$. 


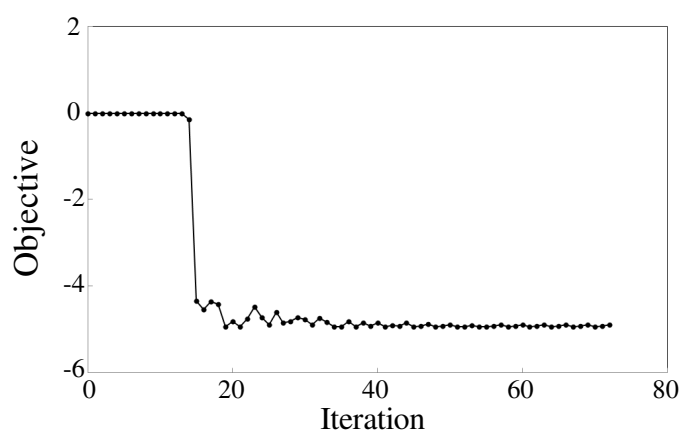

(a)

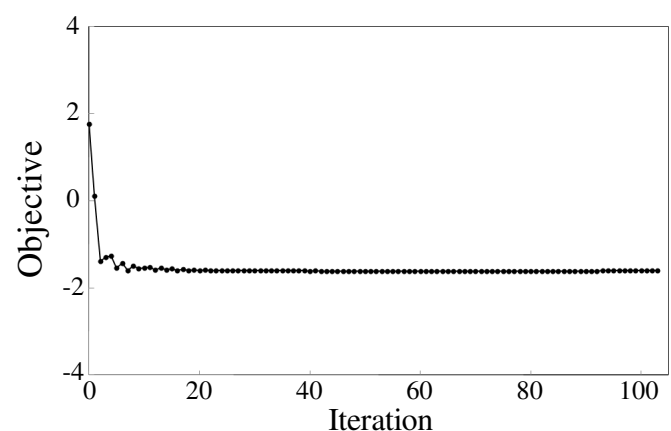

(b)

Fig. 35. Convergence histories of objective function for three-dimensional effective permeability minimization problem targeting $0.45 \mathrm{THz}$ : (a) $1^{\text {st }}$ optimization stage; (b) $2^{\text {nd }}$ optimization stage. 